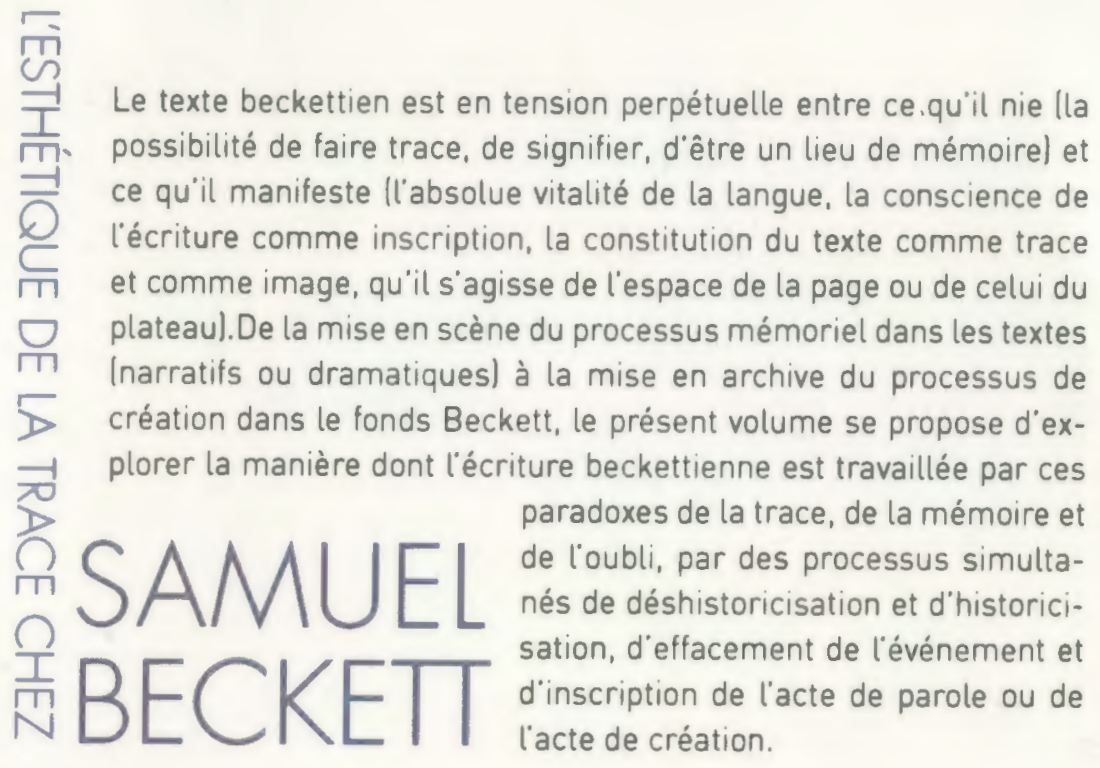

Delphine Lemonnier-Texier est maître de conférences au département d'anglais létudes shakespeariennes et théâtre anglophonel de l'université de Rennes 2 - UEB. Geneviève Chevallier est maître de conférences en études anglophones et membre du CRHI, uiversité de Nice-Sophia Antipolis.

Brigitte Prost est maître de conférences en études théâtrales à Rennes 2- Université européenne de Bretagne.

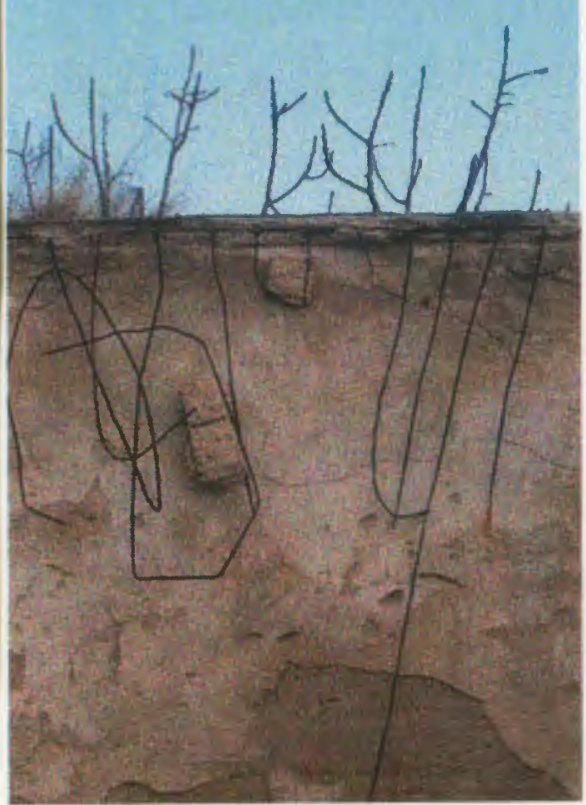

Sous la direclion de Delphine Lemonnier Texier, Geneviève Chevallier et Brigitte Prost

\section{L'ESTHÉTIQUE}
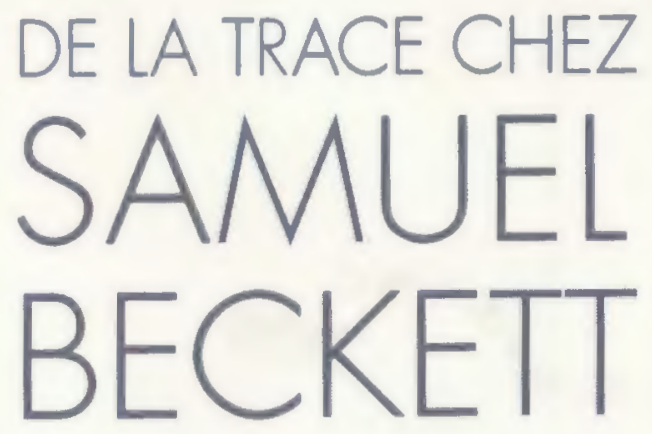

Écriture, représentation et mémoíre

En couverture : Photo de Geneviève Chevallier 


\title{
MISES EN SCENNE ET EN TEXTE DE LA MÉMOIRE DANS CETTE FOIS
}

\author{
Carla TABAN
}

Au moins depuis Proust, l'œuvre de Samuel Beckett explore dans toutes ses dimensions génériques, de manière constante et à de multiples niveaux la problématique de la mémoire. La plupart des études critiques qui portent sur cette problématique la traitent néanmoins, le plus souvent, de façon unidimensionnelle en se penchant seulement sur l'une ou l'autre des trois " "couches" de mémoire " qui constituent, selon Bruno Clément, tout texte beckettien : " une mémoire intertextuelle, une mémoire autotextuelle [et] une mémoire individuelle ${ }^{1}$ ".

Cette fois, dramaricule éminemment exemplaire de ce qu'il est convenu d'appeler le " théâtre de la mémoire " de Becketr ${ }^{2}$, ne jouit pas d'un traitement critique sensiblement différent. C'est pourquoi la présente contribution vise un double objectif. D'une part, nous voulons mettre en évidence d'autres " couches de mémoire " qui opèrent dans Cette fois - notamment " une mémoire scénique ", "une mémoire intratextuelle " et " une mémoire d'autotraduction " - et ains rendre justice à la complexité de cette courte pièce qui apparaît trompeusement

1. Clément Bruno, L'ơuvre sans qualités: rhétorique de Samuel Beckett, Paris, Le Seuil coll. « Poérique ", 1994, p. 353.

2. P'our la discussion la plus informée de Cette fois comme " memory play " "pièce mémorielle ") voir KOZDON Sabine, Memory in Samuel Beckett's Plays. A Psychological Approach, Münster, Lit Verlag, 2005, p. 189-190 et 213-231. Kozdon observe que beaucoup de critiques emploient le rerme de "memory play " par rapport à des pièces théâtrales, radiophoniques ou télévisuelles de Beckett sans toutefois le définir préalablement. Selon les critères de Kozdon, qui articule le " théâtre de la mémoire " de Beckett autour de la " mémoire individuelle " de Clément, seulement Comédie, Pas moi er Cette fois sont des "exemples exceptionnellement purs " des " pièces mémorielles " becketriennes. 
simple. D'autre part, et en même temps, il s'agira de montrer comment les diverses " couches de mémoire " qui configurent Cette fois ne fonctionnent pas isolement, mais interagissent et se conditionnent réciproquement.

\section{Cette fois: genèse, mise en scène, autotraduction et publication}

Pour situer notre propos, il convient de commencer avec une brève "fiche signalétique » du dramaticule, qui enregistre les données factuelles de sa genèse, mise en scène, autotraduction et publication. Beckett rédige Cette fois, en anglais, entre le début juin 1974 et août $1975^{3}$. La première de That Time (metteur en scène: Donald McWhinnie; comédien: Patrick Magee), qui a lieu au Royal Court Theatre de Londres au printemps 1976, repose sur des notes de mise en scène préparées par l'auteur à l'intention de McWhinnie. Faber \& Faber publient l'édition princeps de That Time à cette occasion. Beckett met lui-même en scène la version allemande de la pièce, Damals (comédien: Klaus Herm), au SchillerTheater Werkstatt de Berlin en automne 1976, en revoyant lors du travail de création du dramaticule au théâtre la traduction en allemand qu'Elmar Tophoven en avait faite auparavant ${ }^{4}$. Cette fois, autotraduction en français de That Time (et Damals), est publiée aux Éditions de Minuit en 1978 et réalisée au Théâtre Gérard Philippe de Saint-Denis en 1983 (metteurs en scène: David Warrilow et Rocky Greenberg; comédien : David Warrilow) ${ }^{5}$.

3. Stanley E. GONTARSKI retrace dans son ouvrage The Intent of Undoing in Samuel Beckett's Dramatic Texts, Bloomington, Indiana University Press, 1985, chapitre 10 " "Making yourself all up again" in That Time ", p. 150-161, les dix étapes manuscrites et tapuscrites de la genèse de That Time et leurs traits de rédaction les plus saillants, dont, notamment, le rravail que Beckett a mené, après avoir élaboré chacune des bribes de voix $\mathrm{A}, \mathrm{B}$ et $\mathrm{C}$ en continuité, pour les fragmenter, réorganiser et complexifier les rapports entre elles.

4. Selon James Knowlson, Beckett trouvait cette traduction insatisfaisante: "The Tophovens' translations of Foorfalls and That Time, needed for a Schiller-Theater production he was to do himself in September [1976], were not, Beckett felt, up to their usual high standard, and he had to hold up their publication with Suhrkamp until they could work on them together. "Cf. KNOWLSON James, Damned to Fame. The Life of Samuel Beckett, New York, Simon \& Schuster, 1996, p. 552 et notes afférenres.

5. Pour les données sur la genèse, mise en scène, autotraduction et publication de Cette fois voir ACKERLEY Chris J. et GONTARSKI Stanley E., The Grove Companion to Samuel Beckett. A Reader's Guide to His Works, Life, and Thought, New York, Grove Press, 2004, p. 568-570; GONTARSKI Sranley E. (dir. ex introd.), The Theatrical Notebooks of Samuel Beckett. Volume IV: The Shorter Plays, with revised texts for Footfalls, Come and Go and What Where, New York, Grove Press, 1999, Part V: That Time, p. 353-404; et CHABERT PIERRE et al. (dir.), Revue d'Esthétique, numéro hors série "Samuel Beckett ", 1990, p. 442.
Le texte de Cette fois, auquel Beckett n'aboutit que suite et grâce aux diverses expériences de création et révision de la pièce en anglais et allemand ${ }^{6}$, porte les " traces" des modifications accompagnant les processus de mise en scène, correction et autotraduction du dramaticule. Ces « traces » forment et transforment les multiples "mémoires " de Cette fois dont chacune constitue le point d'ancrage d'une des sections suivantes.

\section{La " mémoire scénique " de Cette fois}

La " mémoire scénique " de Cette fois comprend ces éléments-là du texte français qui sont absents des textes anglais (That Time) et allemand (Damals) ou qui sont différents par rapport à eux, et dont la raison d'être se laisse retracer - grâce aux notes de mises en scène rédigées par Beckett - à des aspects concrets et précis de la création du dramaticule au théâtre. Les " traces » de la "mémoire scénique " de Cette fois se retrouvent donc principalement dans les didascalies de la pièce et portent sur les diverses composantes de son dispositif scénique?

On retrouve un exemple complexe qui relève (aussi) de la "mémoire scénique " du dramaticule dans la toute première didascalie de Cette fois qui précise: "Vieux visage blême légèrement incliné en arrière, longs cheveux blancs dressés comme vus de haut étalés sur un oreiller " (p. 9), tandis que That Time propose: "Old white face, long flaring white hair as if seen from above outspread " (p. 6) et que Damals donne: "Altes, weisses Gesicht, Mähne nach oben ausgebreiteten weissen Haars wie das eines Liegenden ${ }^{8}$. „ (p. 7) En ajoutant, par rapport à

6. Knowlson laisse entendre que Beckett a travaillé sur l'autotraduction en français de That Time pendant la révision de Damals et la préparation de la mise en scène de Berlin (KNOWLSON James, Damned to Fame, op. cit., p. 552), tandis que John Pilling suggère que Beckett a commencé l'autotraduction de Cette fois en novembre 1976, donc peu après la première de Damals: PILLING John, A Samuel Beckett Chronology, Houndmills, Basingstoke, Hampshire, New York, Palgrave, Macmillan, 2006, p. 201

7. le dispositif scénique du dramaticule comprend: 1) une dimension visuelle concrétisée dans l'image de la tête du seul et unique personnage de Cette fois, qui " flotte " littéralement dé-corporalisée, dans le noir, à trois mètres au-dessus de la scène et un peu décentrée; et les changement d'éclairage dont cette image fait l'objer ; 2) une dimension auditive concrétisée dans: les enregistrements acousmatiques des bribes de voix A, B et C qui accompagnent l'image de la tête; leu interruption deux fois au cours de la pièce; et la respiration du personnage présent sur scène; 3 , une dimension motrice concrétisée dans la nimique du protagoniste (ouverture et fermeture des yeux, sourire final édenté) ; et 4) une dimension dynamique concrétisée dans la corrélation des trois dimensions précédentes.

8. Vuir BECKETT Samuel, That Time / Damals, édition bilingue, traduction en allemand de Elmar Tophoven, Frankfort sur le Main, Suhrkamp Verlag, 1976; et idem, Cette fois, in Catastrophe et autres dramaticules (C'ette fois, Solo, Berceuse, Impromptu d'Ohio), l'aris, Éditions de Minuit, 1982, p. 7-25 
That Time et Damals, que le visage du "Souvenant " est "légèrement incliné en arrière " et que ses cheveux sont " comme [...] étalés sur un oreiller ", Cette fois accentue la coordonnée horizontale de la perspective composite qui caractérise l'image scénique du dramaticule, en lui prêtant autant d'importance qu'à la coordonnée verticale qui étair, elle, bien présente dès That Time: "face about ten feet above stage level [...] as if seen from above " (p. 6) / "visage [...] à environ 3 mètres au-dessus du niveau de la scène [...] comme [vu] de haut » (p. 9).

Dans Cette fois l'impossible simultanéité de deux points de vue opposés selon lesquels le spectateur perçoit le visage du "Souvenant " de bas, mais comme s'il le regardait de haut - est renforcée par l'emphase sur le renversement de la tête du protagoniste, dont l'horizontalité est supposée faciliter la construction, voire la distorsion, du regard montant du spectateur comme regard plongeant. Damals, étape intermédiaire entre That Time et Cette fois, enlève toute mention relative à la vue en bas du public, que Cette fois récupérera par la suite et que la version allemande compte accomplir indirectement en commençant justement par rendre plus explicite la position horizontale du " Souvenant » dont le visage et les cheveux sont comparés à " ceux de quelqu'un qui est étendu / couché " vraisemblablement sur le dos?.

Le fait est que la "mémoire " en jeu dans cet exemple est en effet (non seulement, mais aussi) une "mémoire scénique " - autrement dit que Beckett a opéré les transformations et ajouts discutés ci-dessus (aussi) à la suite de son expérience de metreur en scène du dramaticule - est appuyé par l'entrée suivante, inscrite dans son cahier de notes berlinois sous la rubrique "Listener's Reactions " : " 4. If hand allowed in image, holding sheet at neck or collar of shirt, it should tighten hold for

9. Plusieurs critiques ont déjà atriré l'attention sur la contradiction perceptuelle que propose l'image scénique de Cette fois, en l'interprérant comme une "expérience faite sur la perspecrive visuelle " et l'« illusion optique » et en soulignant son " irréalité " qui désoriente spatialement le spectateur. On a aussi proposé de possibles sources d'inspiration artistiques pour cette image, qui vont des gravures à sujet ancien testamentaire de William Blake à certaines roiles de Salvador Dali représentant des têtes dé-corporalisées, en passant par la photo "Femme aux longs cheveux " (1929) de Man Ray. Voir à ce sujet BRATER Enoch, Beyond Minimalism: Beckett's Late Style in the Theatre, Oxford, Oxford University Press, 1990, chapitre 3 " That Time on That Space ", p. 37-51; GONTARSKI S. E., The Theatrical Notebooks of Samuel Beckett. Volume IV: The Shorter Plays, op. cit., note 5, p. 393; KNOWLSON James, Damned to Fame, op. cit., p. 531 ; et MCMULLAN Anna, "Samuel Becketr's Cette fois: Between Time(s) and Space(s)", French Studies: A Quarterly Review, vol. $44, n^{\circ} 4$, ocrobre 1990, p. 424-439. Ces criricues omertent toutefois de remarquer que les versions anglaise, allemande et française n'offrent pas exactement la même image scénique ou, mieux dit, que cette image n'est précisée dans toute son impossibilité percepruelle que progressivement, d'une étape à l'autre d'(auto)traducrion et de création de la pièce. silence, relax for listening ${ }^{10}$. "Bien que l'image de la main n’ait pas été finalement retenue, à côté de celle de la tête, dans la production autorisée de Damals à Berlin, le fait que cette main aurait pu serrer, dans une des possibilités envisagées, un drap près du cou, suggère que Beckett a considéré assez sérieusement l'alternative de fournir aux spectateurs - par le biais de l'accessoire du drap - des indices précis sur l'horizontalité du "Souvenant » et donc de montrer effectivement celui-ci sur scène dans la posture de quelqu'un qui est alité, posture que la didascalie allemande mentionne, mais seulement comme terme de comparaison (conceptuelle) de la position du personnage et non comme une réalité scénique (concrète).

L'« oreiller " de Cette fois est une " trace mémorielle " métonymique qui spécifie, voire concrétise, le terme de comparaison allemand de Damals ${ }^{11}$ et il est en même temps une " trace mémorielle " isotopique du " drap " éventuel de la note de mise en scène citée. Encore faut-il remarquer que cet " oreiller " relève non seulement d'une "mémoire d'(auto)traduction" (qui rappelle à la fois l'anglais That Time et l'allemand Damals comme précurseurs de Cette fois) et d'une " mémoire de la représentation théâtrale " (qui convoque les notes beckettiennes de mise en scène), mais aussi d'une " mémoire génétique »: "From the earliest manuscript through the heavily revised fifth typescript lof That Time], Beckett had the head resting on a pillow. This early image repeats one from the discarded versions of "Kilcool", but Beckett [revised it to] "No pillow"12."

Les divers types de " traces mémorielles " qui convergent et œuvrent dans cet exemple connotent Cette fois non pas comme un texte achevé une fois pour toutes, mais comme un site en processus de passage - de la page à la scène et viceversa -, d'une langue à l'autre, de même qu'entre des stades génétiques non-consécutifs $^{1,3}$-, site qui configure avec d'autres sites en processus beckettiens l'œuvre

10. GUNTARSKI Stanley E., The Theatrical Notebooks of Samuel Beckett. Volume IV: The Shorter Plays, op. cit., p. 373, 375

11. L'« oreiller " de " longs cheveux blancs dressés comme vus de haut étalés sur un oreiller " (Cette fois) esc une concrérisation de " tignasse de cheveux blancs dressés comme ceux de quelquiun qui est étendu/couchée " (Damals, notre traduction et emphase) dans la mesure où la version française se laisse lire de deux nanières: comme "cheveux [...] comme [...] éralés sur un oreiller ", ainsi que nous l'avons proposé plus haur, mais aussi comme "cheveux [...] étalés sur un oreiller " donc de manière à donner une matérialité d'accessoire à l'" oreiller ".

12. GONTARSKI Stanley E., The Intent of Undoing in Samuel Beckett's Dramatic Texts, op. cit., p. 155.

13. Il s’agit de "stades génériques non-consécurifs" dans le sens où Cette fois maintient par endroits (voir le terme de comparaison de l'" oreiller") des rapports plus étroirs avec la créarion de Damals, le texte de Damals et certains avant-texres de That Time qu'avec That Time lui-même C'est comme si le texte publié anglais, qu'on considérerait normalement comme la condition sine qua non de route autotraduction ou créarion du dramaricule, n'était en réalité qu'un châ̂- 
modulaire de l'auteur. Les « traces " que That Time, Damals, leur genèse, création et (auto)traduction inscrivent dans (l'" oreiller " de) Cette fois connotent donc des significations plus ou moins analogues à celles qu'on attribue souvent à la structure et surtout au contenu thémarique mnémonique de ce dramaticule: la simultanéité différentielle des bribes de voix $A, B$ et $C$, mais aussi des " souvenirs " que chacune d'entre elles évoque, leur multiplicité, hétérogénéité, fragmentation, redondance, possibilité, non-linéarité, inconséquence et inachèvement.

Il convient toutefois de remarquer que l'intérêt de Cette fois réside autant, sinon plus, dans les modalités textuelles et scéniques particulières employées pour mettre en place les diverses " traces mémorielles " du dramaticule et leur faire connoter les significations citées, que dans ces seules connotations elles-mêmes. D'autres didascalies de la version française, qui gardent elles aussi la "mémoire " des mises en scènes autorisées antérieures de la pièce, permettent de mieux préciser, avant toute signification connotative qu'on pourrait leur attribuer, leur constitution et fonctionnement "à la limite même de ce qui est possible au théâtre ${ }^{14}$ ".

Cette fois contient plusieurs didascalies très brèves dictant de légères montées et baisses de l'éclairage sur le visage du "Souvenant ", là où That Time et Damals n'en disent rien. Ces ajouts didascaliques sont sûrement dus à l'expérience de Beckett comme conseiller de Donald McWhinnie pour That Time à Londres et comme metteur en scène de Damals à Berlin, puisque la plupart des notes qu'il a préparées à ces occasions portent en effet sur des changements d'éclairage et sur leur corrélation avec des changements concernant les bribes de voix enregistrées, la respiration du protagoniste et sa mimique ${ }^{15}$. Cette fois n' incorpore néanmoins, avec ses quelques observations laconiques sur l'éclairage, qu'une infime partie des notes de mise en scène beckettiennes, qui permettent en effet de reconnaître le patron général d'opération : "À l'approche du silence monter l'éclairage. Quand

non dispensable du devenir de Cette fois (et Damals). Ce phénomène de l'origine inessentielle ou relative caractérise aussi l'autotraduction becketrienne lorsqu'elle procèdè à partir des étapes génétiques antérieures au texte source publié ou à partir des stades historiques antérieurs de la langue source.

14. C'est Beckett lui-même qui aurait affirmé que Cette fois se situe " on the very edge of what is possible in the theatre». Voir KNOWLSON James, Damned to Fame, op. cit., p. 533 et note 162, p. 731, notre traduction.

15. À Berlin (mais non à Londres) les bribes de voix non seulement s'interrompent et recommencent, mais elles s'affaiblissent et ralentissent aussi progressivement, pour revenir par la suite à leur débir et volume initiaux. La respiration régulière du proragoniste n’est pas elle non plus simplement audible ou non, mais elle est soumise elle aussi à des variations graduelles de volume les réminiscences recommencent le baisser ${ }^{16}$ ", mais qui ne suffiraient point à un metteur en scène de Cette fois pour créer un spectacle comparable au That Time de McWhinnie, ni, surtout, au Damals de Beckett.

Du printemps à l'automne 1976, Beckett révise la manière précise dont interagissent les changements affectant l'éclairage et les autres dispositifs scéniques. Dans toutes les versions des rextes publiés (That Time, Damals et Cette fois) la représentation théâtrale du dramaticule commence avec une scène dans l'obscurité où la montée de l'éclairage découvre le visage aux yeux ouverts du "Souvenant " dont la respiration est audible. L'image est tenue, selon les textes, sept secondes avant que l'enregistrement sonore de la voix ne soit déclenché. Une fois que la bribe de voix $\mathrm{A}$, la première à intervenir, finit de dire " cette fois où tu es retourné cette dernière fois voir si elle était là toujours la ruine où enfant tu te cachais quand cétait ", une didascalie indique que les yeux du "Souvenant "se ferment et, dans Cette fois (mais non dans That Time et Damals), que l'éclairage baisse: " les yeux se ferment, légère baisse de l'éclairage " (p. 9). Dans ses notes londoniennes, Beckett donne plus de détails: l'éclairage commence à baisser peu après la fermeture des yeux sur "quand c'était " - notamment à partir de " on from there " (p. 8) / "de là à pied " (p. 10) - et il atteint son minimum d'intensité, équivalant à la moitié de l'intensité initiale, à la fin de cette intervention de A: "when was that » (p. 8) I "quand c'était" (p. 10)

Dans les textes publiés français, anglais et allemand le changement suivant du dispositif scénique, qui a lieu après douze interventions de $A, B$ et $C$ (dans l'ordre $A_{1} C_{1} B_{1} / A_{2} C_{2} B_{2} / A_{3} C_{3} B_{3} / C_{4} A_{4} B_{4}$ ), est décrit didascaliquement ainsi: "Silence 3 secondes. Les yeux s'ouvrent. Légère montée de l'éclairage. Respiration audible. 7 secondes " (p. 14) / "Silence 10". Breath audible. After 3" eyes open " (p. 30) / "Stille 10". Hörbares Atmen. Nach 3" Augen [geöfnet] öffnen sich $^{17}$ " (p. 31). Cette fois est de nouveau le seul texte parmi les trois à mentionner une montée de l'éclairage, qui suit l'arrêt de la voix et vraisemblablement aussi l'ouverture des yeux du "Souvenant ". Les notes pour McWhinnie different sensiblement de Cette fois à cet égard: l'éclairage commence à monter non pas après que B s'arrête, mais pendant sa dernière intervention dans cette première partie du dramaticule - à partir de " to believe " (p. 30) / "à croire [que tu aies

16. GONTARSKI Stanley E., The Theatrical Notebooks of Samuel Beckett. Volume IV: The Shorter Plays, op. cit., p. 369, 371, nous traduisons.

17. L'édition bilingue de That Time / Damals que nous utilisons est celle que Becketr lui-même a employée et corrigée à Berlin. Les mots [barrés] entre crochets représentent ses révisions du texte allemand du dramaticule. Cf. GONTARSKI Stanley E., The Theatrical Notebooks of Samuel Beckett. Volume IV: The Shorter Plays, op. cit., p. 397-404 
jamais pu...]» (p. 14) - et il atteint son intensité originale avec la fin de ce fragment. Si les notes de Londres montrent Beckett hésitant sur les mots particuliers où un changement d'éclairage commence à accompagner l'enregistrement, elles indiquent clairement le fait que ce changement a toujours lieu simuiltanément avec la voix enregistrée et non tantôt sur fond sonore, tantôt sur fond de silence comme le suggère Cette fois.

Les notes berlinoises élaborent encore plus la synchronisation des changements scéniques initiée quelques mois plus tôt dans les notes londoniennes, dont Cette fois ne garde que des " traces mémorielles " imprécises et concises, et dont les versions publiées de That Time et Damals ne gardent textuellement aucune "mémoire ". Pour sa propre création du dramaticule, Beckett coordonne l'enregistrement et l'éclairage non seulement entre eux, mais aussi avec la mimique er la respiration du "Souvenant ". Il varie aussi la qualité des bribes de voix à l'approche des silences. Un cadre temporel plus rigoureux et régulier de dix secondes est dressé pour la tenue d'une image et/ou l'établissement de nouveaux repères lexicaux indiquant l'initiation et l'accomplissement d'un changement. Le tableau qui suit résume les différences entre le texte publié de Cette fois et les notes de mise en scène beckettiennes pour That Time et Damals ${ }^{18}$.
Londres (printemps 1976)

Ouverture: Érablir le visage en plein éclairage, yeux

ouverts.

$A_{1}:$ P'eu après la fermeture des yeux, indice: "de lia à pied » (p. 10), commencer la baisse de l'éclairage à moirié de l'intensité iniriale, atreince à la fin du fragment avec " quand c'était " (p. 10)

$\mathrm{B}_{4}$ : Monter l'éclairage à partir de [तांuretamouraे quetqưun"] "à croire " (p. 14) jusqu'à atteindre l'intensité initiale à la fin du fragment avec "suaire" (p. I4)

Peu après la fermeture des yeux, indice: [त्टाe fois"] "qu'un seul" baisse de l'éclairage à moirié de l'intensité initiale, atteinte à la fin du fragment avec " une autre fois " (p. 15) (p. 15), commencer

Berlin (automne 1976)

Monter l'éclairage au maximum en ca. 10 " sur le visage aux yeux ouverts. Tenir $10^{\prime \prime}$

: Avec " certe fois " $(p .9)$ commencer la baisse de

l'éclairage au minimum,

atteint en ca. I()" avec

"quand c'était » (p. 9) er la fermerure des yeux.

$\mathrm{B}_{4}$ : Avec " pour contenir le vide " (p. 14) commencer la montée de l'éclairage au maximum, atteint en ca. 10 " avec " suaire" (p. 14) et l'ouverture des yeux. Tenir 10". Ralentir et affaiblir la voix à partir de “ jusqu'à n'y voir" (p. 14).

$\mathrm{C}_{5}$ : Avec " jamais le même " (p. 15) commencer la baisse de l'éclairage au minimum, atteint en ca. 10"

avec « allons» (p. 15) er la fermeture des yeux. Débit et

. Le tableau sélectionne, réorganise, synthétise, présente dans un autre format et traduit en français ces notes, dont le facsimile et la transcription peuvent être consultés dans GONTARSKI S. E., The Theatrical Notebooks of Samuel Beckett. Volume IV: The Shorter Plays, op. cit., p. 358-363 er 368-391.Les mots [barrés] entre crochets continuent à représenter les correcrions de Beckett, notamment les choix d'indices dictant un changement scénique qu'il a éliminés et remplacés avec d'autres. Les mors soulignés simplement représentent les indices d'éclairage de Londres. Les mots soulignés doublement représentent les indices d'éclairage et ceux soulignés de manière renforcée représentent les indices de voix de Berlin. Les barres obliques / délimitenr, à l'intérieur d'un seul et même fragment, le passage d'une page à la suivante dans l'édition de Cette fois utilisće.

\section{Cette fois (1978)}

"Rideau. Scène dans l'obscurité. Montée de l'éclairage sur le visage du Souvenant [...]. Silence 7 secondes. Yeux ouverts. Respiration audible, lente et régulière." (p. 9)

\section{$A_{1}:$ " cette fois où tu es} retourné cette dernière fois voir si elle érait là toujours la ruine où enfant tu te cachais quand c'étair (les yeux se ferment, légère baisse de l'éclairage) journée grise avec le 11 jusqu'au bout de la / ligne et de là à pied ... " (p. 9-I0).

$B_{4}:$ : $[\ldots]$ et peu à peu difficile à croire de plus en plus difficile à croire que tu aies jamais pu jurer amour à quelqu'un ou jamais quelqu un à toi usauàny que tu allais inven contenir le vide [...] Silence 3 secondes. Les yeux souvrent. Légère montée de l'éclairage. Respiration audible. 7 secondes" ( $p .14)$

$\mathrm{C}_{5}$ : " jamais le même mais le même que qui bon Dieu '́es-tu jamais dit je de ta vie allons (Les yeux se ferment. légère baisse de l'éclairage) as-tu jamais pu te dire je de ta vie tournant voilà un mot que tu avais toujours a la bouche avant qu' elle tarisse pour de bon toute ta vie dans les tournants l'un / après l'autre alors que jamais qu'un seul $[. .$.$] " (p. 14-15).$ 
$A_{8}$ : Monter l'éclairage à partir de [थ्कiोtrétaism] "ne sachant plus" (p. 19) jusqu'à atteindre l'intensité iniriale à la fin du frag-

ment avec " nul ne venait " (p. 19).

A.: Avec " seul au monde" (p. 19) commencer la montée de l'éclairage au maximum, atteint en ca. 10 " avec "nul ne venait (p. 19) et l'ouverture des yeux. Tenir 10". Ralentir et affaiblir la voix à partir de " ni quand" (p. 19).

$B_{3}$ : Peu après la fermeture des yeux, indice: " avec les mules fantômes " (p. 20), commencer la baisse de l'éclairage à moitié de l'intensité iniriale, atteinte à la fin du fragment avec " tout avec" (p. 20).

$\mathrm{A}_{12}$ : Monter l'éclairage à partir de " remonter au bord " (p. 24) jusqu’à atreindre l'intensité iniriale à la fin du fragment avec

"ne jamais revenir (p. 24).

: Avec " ou seul dans les mêmes scènes " (p. 20) commencer la baisse de l'éclairage au minimun, atteint en ca. 10" avec

"sur la pierre" (p. 20) et la fermeture des yeux. Débit volume initiaux de la voix.

$\mathrm{C}_{12}$ : Avec "quelque chose comme ça " (p. 24) commencer la montée de l'éclairage au maximum, atteint en ca. 10 " avec "venu parti " (p. 24) et l'ouverture des yeux. Tenir 10 ". Ralentir et affaiblir la voix a partir de " sinon qu'est-ce qu'elle t'a dit " (p. 24)

Baisse finale de l'éclairage. Baisser l'éclairage ca. 10"

Nores berlinoises sur les réactions du " Souvenant "

- La Respiration: monte avec la montée de l'éclairage, est audible pendant les silences et les yeux ouverts, baisse à inaudible avec la baisse de l'éclairage et la reprise de la voix.

- Lors de l'éclairage maximal final: 5" seuls yeux ouverts, puis 5" yeux ouverts er sourıre: celui-ci continue pendant la baisse finale de l'éclairage. sachant plus où tu étais ni.
$A_{8}:$ : $[\ldots]$ ne sachant plus où tu étais peu à peu ne guand ni pourquoi sem all monde pour autant que tu saches comme cette fois $[\ldots]$

Silence 3 secondes. Les yezix souvrent. Légère montee de l'éclairage. Respiration audible. 7 secondes." ( $\mathrm{p} .19)$

$B_{9}:$ " wuseul dans les mêmes scènes l'inventant ainsi histoire de tenir contenir le vide surla pierre (les yeux se ferment, légère baisse de l'éclairage) seul au blés l'azur ou le halage seul sur le halage avec les mules fancômes $[\ldots] »($ p. 19-20).

$C_{12}$ : " [...] pas un bruit sinon_un'est-ce unielle ća dit venu parti est-ce que c'étai ça velque chose comme personne venu personne peine venu parti.

Silence 3 secondes. Les yeux souvrent. Légère montée de l'éclairage. Respiration andible. Sourire, édenté de préférence. 7 secondes. (p. 24).

"L'éclairage séteint lentement. Rideau. $)($ p. 24)
Dans la mise en scène de Beckett la montée de l'éclairage sur le visage du "Souvenant" au tout début de la pièce dure dix secondes et une fois que celui-ci atteint son intensité maximale, l'image est tenue dix autres secondes (et non sept comme le précisent les textes publiés). L'éclairage commence à baisser simultanément avec le déclenchement de l'enregistrement sonore acousmatique, c'est-à-dire avec les tout premiers mots de $A_{1}$ - " cette fois où tu es retourné... " (p. 9) - et il atteint son minimum non pas à la fin de cette intervention de $\mathrm{A}$ (comme dans les notes de Londres), mais en même temps que les yeux du "Souvenant " se ferment sur le premier " quand c'était » (p. 10) de la pièce, c'est-à-dire après environ dix secondes. Le changement suivant a lieu lors de $\mathrm{B}_{4}$ : l'éclairage commence à monter avec "pour contenir le vide" (p. 14) - donc plus tard que dans les notes pour McWhinnie - et il atteint son intensité maximale initiale après environ dix secondes, à la fin du fragment, au moment même où le "Souvenant " ouvre les yeux sur "suaire" (p. 14). L'image est tenue pendant dix secondes avant que l'éclairage ne commence à baisser de nouveau, simultanément avec la reprise de la voix $\mathrm{C}_{5}$ " jamais le même " (p. 15) et qu'il n'atteigne son intensité minimale en dix secondes, sur " allons ", indice qui dicte aussi la fermeture des yeux du "Souvenant". Ce patron de mise en scène qui complexifie celui de Londres est poursuivi régulièrement tout au long du dramaticule.

À la différence des notes londoniennes et des textes publiés, qui n'en font aucune mention, les notes berlinoises concernant les réactions du "Souvenant " précisent que les interruptions de la voix après $B_{4}, A_{8}$ et $C_{12}$ sont précédées par son ralentissement et affaiblissement, initiés - comme les repères lexicaux le montrent - avant la montée de l'éclairage. Lors de ses reprises par $\mathrm{C}_{5}$ et $\mathrm{B}_{9}$ la voix relève d'un volume et débit identiques à ceux du début du dramaticule. La respiration du "Souvenant » est elle aussi variable à Berlin. Son audibilité monte en même temps que la montée de l'éclairage, atteint son maximum avec lui, accompagne les intervalles de silence pendant lesquels les yeux du "Souvenant " son ouverts et redevient inaudible avec la baisse de l'éclairage et la reprise de la voix.

La synchronisation des diverses dimensions du dispositif scénique que Beckett opère à Berlin œuvre, selon lui, à abolir tout rapport de causalité que le spectateur serait enclin à établir entre elles à la suite de l'application du principe fallacieux post hoc ergo propter hoc. Puisque le "Souvenant" ouvre les yeux, au Schiller-Theater Werkstatt, simultanément avec l'arrêt de la voix, "it is not decided whether he opens his eyes and the voice stops for that reason or whether the voice stops and therefore he 
opens his eyes ${ }^{19}$ ". Toujours est-il que la synchronisation proposée reste (délibérément?) imparfaite et que le décalage temporel entre certains changements continue à favoriser leur interprétation causale. Cela est surtout vrai pour les modifications concernant les " ritardando ${ }^{20}$ " de l'enregistrement dans $\mathrm{B}_{4}, \mathrm{~A}_{8}$ et $\mathrm{C}_{1,}$ qui commencent avant la montée de l'éclairage et suggèrent ainsi la possibilité que ce sont en effet le ralentissement et l'affaiblissement de la voix qui dérerminent tous les autres changements subséquents.

Parallèlement avec le souci d'une coordination généralement plus étroite entre les différents aspects du dispositif scénique, la mise en scène de Berlin prend aussi beaucoup plus de soin que celle de Londres à éliminer de la scénographie tous grands contrastes surprenants, comme celui concernant la montée finale de l'éclairage - qui atteint son maximum à la fin de $\mathrm{A}_{1}$ à Londres et à la fin de $\mathrm{C}_{1}$ à Berlin. Au Royal Court Theatre, l'intervention $\mathrm{C}_{12}$ de la voix est singularisée par le fait qu'elle est la seule à avoir lieu en plein éclairage. La similitude d'éclairage entre $\mathrm{C}_{12}$ et les intervalles de silence qui la précèdent et la suivent dans le dramaticule convie le spectateur à trouver une connexion analogique, causale ou même chronologique entre la voix $\left(C_{12}\right)$ et le silence, des termes habituellement antithétiques. La régularité, voire l'uniformité, des changements qui accompagnent $\mathrm{B}_{4}, \mathrm{~A}_{8}$ et $\mathrm{C}_{12}$ dans la création autorisée de Damals vise par contre, comme nous l'avons vu, à enlever la plupart des conditions favorables à l'établissement de quelque rapport préférentiel que ce soit entre les diverses dimensions du dispositif scénique, qui semblent ainsi soit simplement juxtaposées, soit capables d'entretenir une multitude non-exclusive de rapports ${ }^{21}$

19. ASMUS Walter D., "Rehearsal Notes for the German Premiere of Beckert's That Time and Footfalls", in Stanley E. GONTARSKI (dir. et introd.), On Beckett. Essays and ('riticism, New York, Grove Press, 1986, p. 335-349, p. 348. (Ces notes ont été publiées originairement dans Journal of Beckett Studies, $n^{\circ} 2$, été 1977, p. 82-95.)

20. "Beckett reminds Herm to remember the ritardando at the end of parts 1, 2, and 3 " (ASMUS Walter D., "Rehearsal Notes ", art. cit., p. 348).

21. Il convient de norer que le sourire final "édenté de préférence " (p. 25) reste, dans les textes publiés, comme dans les mises en scène autorisées du dramaticule, un élément de surprise constant que la critique a interprété de maintes façons. Antoni Libera propose par exemple que le "Souvenant " sourit à la fin de la pièce parce que l'ordre $B_{12} A_{12} C_{12}$ - qui devrait changer lors des dernières interventions des bribes de voix à $\mathrm{A}_{12} \mathrm{~B}_{12} \mathrm{C}_{12}$, en accord avec les règles mises en place par le dramaticule lui-même, mais qui ne change en effet pas - représente la chronologie correcre des souvenirs de jeunesse (B), de maturité (A) et de vieillesse (C). S. E. Gontarski comprend le sourire du "Souvenant " comme l'expression de son contentement purement formel devant la constance de l'ordre BAC tout au long de la troisième partie du dramaticule, tandis que James Knowlson et John Pilling suggèrent toute une série de possibilités: " [...] The smile comes as a considerable dramatic surprise. Enigma remains, in fact, an essential purt of (its) dramutic effect
Quelle "mémoire scénique " le texte de Cette fois garde-t-il de toutes les indications scénographiques et de leurs transformations lors des créations autorisées de la pièce? De toute évidence une " mémoire "fragmentaire, impossible à reconstruire dans tous ses détails à partir de très peu nombreuses " traces " qu’elle inscrit textuellement, à savoir les quelques didascalies sur la position du "Souvenant » et l'éclairage. Le rôle de ces " traces " n'est pas tant de faciliter, à elles seules, la récupération des mises en scène autorisées du dramaticule, voire d'assurer leur transmission fidèle, mais plutôt de simplement signaler leur existence. Les différences didascaliques entre That Time, Damals et Cette fois invitent le lecteur à supposer que ces textes ne représentent pas les seules étapes par lesquelles le dramaticule est passé, mais qu'il y en a d'autres, non pas textuelles, mais théâtrales. Ces étapeslà ne sont pourtant pas proposées par Cette fois comme un passé plus récent er donc plus important que les textes anglais et allemand, qu'il aurait transformés irréversiblement et auxquels il se serait substitué définitivement. Le texte de Cette fois est beaucoup plus proche, en ce qui concerne les didascalies, de That Time et Damals, que des notes de Londres et Berlin, bien que celles-ci le précèdent chronologiquement de manière plus immédiate. Cette fois ne transmet donc pas ses propres passés textuels et théâtraux complètement, fidèlement, chronologiquement et fixement, mais les signale seulement par des bribes didascaliques. La " mémoire scénique " du dramaticule ressemble dans son type de transmission à la mémoire qu'il configure thématiquement, structurellement et métadiscursivement.

Cette fois transmet aussi, en pointant vers les notes beckettiennes de Londres et Berlin, des " passés scéniques " possibles ou fictifs, dans le sens où ils n'ont pas été effectivement réalisés, mais existent seulement comme des possibilités envisagées de mise en scène. Nous avons déjà cité la suggestion abandonnée d'une image de la main serrant un drap ou le col d'un habit près du cou, à laquelle on peut ajouter deux autres: celle d'une légère agitation du "Souvenant " accompagnant

[...]. Is it here simply a smile of satisfaction at the restoration of these old times? A smile of relief and contentment that at last all the torment is nearly over? A wry reflection on the insignificance of the individual human existence in the context of infinity? Or a smile indicating that even capitulation to the void can still be endured with serence acceptance?" (Voir LIBERA Antoni, "Reading That Time ", in DAVIS Robin J. er BUTL.ER Lance St J. (dir. er introd.), "Make Sense Who May ": Essays on Samuel Beckett's Later Works, Gerrards Cross, C. Smythe, 1988, p. 91-107, p. 91 GONTARSKI Stanley E., "Making yourself all up again" in That Time", art. cit., p. 158; er KNOWLSON James et PILLING John, Frescoes of the Skull. The Later Prose and Drama of Samuel Beckett, Londres, John Calder, 1979, p. 210.) Ces nombreuses interprérations, dont aucune n'est définitive, témoignent de l'ambigü̈ré du sourire du "Souvenant " comme élément scénographique que l'uniformité des autres éléments ne fait que mieux mettre en évidence, mais seulement comme signe à signification(s) indéfinie(s). 
la montée de l'éclairage et le "ritardando " de l'enregistrement avant les silences; de même que celle de l'accompagnement de la montée initiale de l'éclairage sur le visage du "Souvenant " par l'enregistrement ${ }^{22}$. Dans ce type de transmission aussi, d'une "mémoire scénique " potentielle ou hypothétique, Cette fois fonctionne analogiquement à la mémoire tachetée d'invention thématisée dans le dramaticule par toutes les trois bribes de voix ${ }^{23}$.

\section{La " mémoire intratextuelle" \\ et la " mémoire d'autotraduction " de Cette fois}

La " mémoire intratextuelle » de Cette fois désigne les éléments récurrents du dramaticule qui opèrent non seulement sémantiquement, au niveau du signifié, en tant que motifs, leitmotivs ou thèmes, mais aussi scénographiquement, voire textuellement, c'est-à-dire au niveau du signifiant visuel, auditif et/ou scriptural de la pièce, dans ses dimensions matérielles, formelles et structurelles. Ces répétitions à la fois conceptuelles et médiatiques inscrivent dans Cette fois des " traces" similaires ou identiques qui renvoient les unes aux autres et s'évoquent réciproquement au cours de la lecture ou de la spectature du dramaticule.

Selon cetre définition, les patrons consistants - d'éclairage, mimique, respiration et qualité de l'enregistrement - que Beckett a élaborés dans ses notes de mise en scène relèvent simultanément d'une " mémoire scénique " dans le sens élaboré dans la section précédente et d'une " mémoire intratextuelle ". Les baisses et montées de l'éclairage sur le visage du "Souvenant " dans l'environnement immédiat du silence, l'ouverture et la fermeture de ses yeux, les " ritardando " des bribes de voix, etc. reviennent au moins deux (sinon trois) fois identiquement au cours de Cette fois, si bien que les deuxièmes (et troisièmes) occurrences de ces éléments pointent vers leurs manifestations antérieures qui les conditionnent et qu'ils conditionnent à leur tour.

La structure de Cette fois - qui organise le dramaticule en trois parties de longueur presque identique à l'intérieur desquelles les trente-six bribes de voix A, B, C se relaient douze fois sans solution de continuité dans des " tercets " qui reviennent pareillement (dans les deux premières parties), voire invariablement

22. Becketr note dans son cahier de notes de Berlin : "Listener's Reactons: $[\ldots \mid$ 3. Perhaps very slight restlessness coming up to silence $\left(B_{4}, A_{\gamma}, C_{1}\right)$ ? ? [...] Speech during the first fade up to max?? GONTARSKI S. E., The Theatrical Notebooks of Samuel Beckett. Volume IV: The Shorter I'lnys. op. ctt., p. 373, 375)

23. Voir $A_{4}, B_{4}, C_{7}, B_{4}$ et $A_{1}$ (dans la troisième partie) - met elle aussi en place les " traces " d'une " mémoire intratextuelle ".

$\begin{array}{lll}\mathrm{I} & \text { II } & \\ \mathrm{A}_{1} \mathrm{C}_{1} \mathrm{~B}_{1} & \mathrm{C}_{5} \mathrm{~B}_{5} \mathrm{~A}_{5} & \mathrm{~B}_{9} \mathrm{~A}_{4} \mathrm{C}_{9} \\ \mathrm{~A}_{2} \mathrm{C}_{2} \mathrm{~B}_{2} & \mathrm{C}_{6} \mathrm{~B}_{6} \mathrm{~A}_{6} & \mathrm{~B}_{10} \mathrm{~A}_{10} \mathrm{C}_{10} \\ \mathrm{~A}_{3} \mathrm{C}_{3} \mathrm{~B}_{3} & \mathrm{C}_{7} \mathrm{~B}_{7} \mathrm{~A}_{7} & \mathrm{~B}_{11} \mathrm{~A}_{11} \mathrm{C}_{11} \\ \mathrm{C}_{4} \mathrm{~A}_{4} \mathrm{~B}_{4} & \mathrm{~B}_{8} \mathrm{C}_{8} \mathrm{~A}_{8} & \mathrm{~B}_{12} \mathrm{~A}_{12} \mathrm{C}_{12}\end{array}$

Cette " mémoire intratextuelle " structurelle permet d'anticiper, au cours de la lecture plus facilement qu'en siruation de spectacle, l'arrivée et la nature des changements dans l'ordre des " tercets " à la fin de la deuxième partie $\left(\mathrm{C}_{7} \mathrm{~B}_{7} \mathrm{~A}_{7} \rightarrow\right.$ $\left.\mathrm{B}_{8} \mathrm{C}_{8} \mathrm{~A}_{8}\right)$ et au début $\left(\mathrm{B}_{8} \mathrm{C}_{8} \mathrm{~A}_{8} \rightarrow \mathrm{B}_{9} \mathrm{~A}_{9} \mathrm{C}_{9}\right)$ et à la fin $\left(\mathrm{B}_{11} \mathrm{~A}_{11} \mathrm{C}_{11} \rightarrow \mathrm{A}_{12} \mathrm{~B}_{12} \mathrm{C}_{12}\right)$ de la troisième partie, anticipations que le dramaticule confirme effectivement dans les deux premières instances, mais seulement pour mieux les infirmer (autrement dit, pour les infirmer plus efficacement) dans la dernière ${ }^{24}$.

Certains critiques suggèrent que la triade structurelle de Cette fois - qui configure trois bribes de voix, trois parties du texte, trois répétitions identiques des " tercets " à l'intérieur de chacune des parties - se réfléchit diégétiquement et thématiquement dans la tripartition des "souvenirs " évoqués par $\mathrm{A}, \mathrm{B}$ et $\mathrm{C}$ : l'arrivée dans la ville (natale?), le renoncement au retour à la ruine de la Folie Fourier faute de moyens de transport et l'attente du rembarquement sur le pas d'une porte dans A; la pierre à l'orée du petit bois, le halage et les dunes comme cadres spatiaux des "scènes " dans B; le Musée des Portraits, le bureau de poste et la bibliothèque municipale comme locaux favorisant des dislocations identitaires dans $C^{25}$. Toujours thématiquement, on a remarqué l'occurrence d'objets remémorés à usage identique comme la pierre plate de la Folie Fourier dans $\mathrm{A}$; la pierre genre meulière dans $\mathrm{B}$; et la dalle de marbre (du Musée des Portraits) dans $\mathrm{C}^{26}$. La similitude fonctionnelle de ces "souvenirs " établit des connexions sémantiques entre eux à l'intérieur de chacune des bribes de voix aussi bien qu'entre les bribes,

24. Le contexte de la "mémoire intratextuelle " structurelle favorise une interprétation du sourire du "Souvenant " conme une réaction déclenchée par les artentes trompées des lecteurs / spectareurs de Cette fois: ceux-ci anticipent à la fin du dramaticule l'ordre $A_{12} B_{12} C_{12}$, mais ils lisent / entendent à sa place, itérativement, $B_{12} A_{12} C_{12}$

25. Voir LIBERA Antoni, "Structure and pattern in That Time ", Journal of Beckett Studies, $n^{\circ}$, automne 1980, p. 81-89, p. 85.

26. Voir Grossman Evelyne, "À la limite... Lecrure de Cette fois de Samuel Beckert ", in L'Angoisse de penser, Paris, Éditions de Minuit, 2008, p. 109-127, p. 115-116. Si l'on prend en compre les autres sièges de Cette fois, tels que le pas de la porte dans $\mathrm{A}$ ou le siège du bureau de poste dans $\mathrm{C}$, cetre tripartition ne fonctionne plus. 
connexions qui relèvent d'une " mémoire intratextuelle » dans le sens où les termes qu'elles relient se rappellent mutuellement.

La critique beckettienne n'a pas manqué de noter non plus les récurrences textuelles - ou plus exactement intratextuelles - des mêmes mots et syntagmes, voire les récurrences des mêmes procédés stylistiques / rhétoriques, dans Cette fois. Les expressions " cette fois ", " dernière fois ", " autre fois " et " quand c'était " - qui actualisent le thème majeur du dramaticule et dont la première, qui fonctionne aussi comme titre de la pièce, est la plus fréquente - sont les plus exemplaires du premier cas de figure cité ${ }^{27}$. Mais des phrases entières, dont certaines contiennent ces expressions ${ }^{28}$, de même que d'autres syntagmes plus définis et moins sujets aux questionnements et modalisations de la voix sont eux aussi repris, identiquement ou par variation topologique et/ou substitutive, à l'intérieur de $A, B$ et $C$ ou entre les bribes de voix ${ }^{29}$. Quand la reprise a lieu (plus ou moins) au début et à la fin d'un seul et même fragment on a affaire à un dispositif stylistique très similaire au cycle ou à l'antépiphore; une reprise au début des fragments successifs crée une figure comparable à l'anaphore; tandis qu'une reprise à la fin d'un fragment et au début du fragment suivant génère une structure analogue à l'anadiplose ${ }^{30}$.

Tout comme les répétitions scénographiques, structurelles et thématiques, les répétitions des mêmes mots, syntagmes et phrases (qu'elles soient stylistiques / rhétoriques ou non) inscrivent dans Cette fois des "traces mémorielles intratextuelles». Le plus souvent la critique se contente de simplement constater ce type de répétitions et, tout au plus, d'en proposer des interprétations connotatives, sans les érudier dans leur particularité et leur fonctionnement propre ${ }^{31}$. Dans ce qui

27. Voir CONNOR Steven, Samuel Beckett. Repetition, Theory and Text, Oxford et New York, Basil Blackwell, 1988, chapitre 6 "Presence and Repetition in Beckett's Theatre ", p. 115-139, p. 137.

28. Cette fois ne contient pas de "phrases" à proprement parler puisque le texte est dépourvu de tour signe de ponctuation er se soustrait ainsi à tour découpage syntaxique figé. "Phrase " est donc à comprendre ici comme une unité syntaxique potentielle er non actualisée/matérialisée effecrivement.

29. Parmi les irérations syntagmatiques et phrastiques moins remarquées par la critique on peut citer: " nuit noire ou clair de lune " $\left(A_{4}, A_{4}, A_{11}, B_{12}\right) ;$ " te rend[re] $[\ldots]$ à l'évidence " $\left(A_{5}, C_{111}\right)$; et "tout droir $[\ldots]$ ni à droite ni à gauche" $\left(A_{2}, A_{5}, C_{4}, A_{12}\right)$.

30. $A_{v}$ illustre un cycle parfait, tandis que les successions $A_{2}-C_{2}$ et $A_{4}-B_{4}$ illustrent une anadiplose et respectivement une anaphore imparfaites.

31. Steven Connor attire l'attention sur le caractère différentiel des répétitions er suggère que celluici empêche d'organiser les "récits" respectifs de A, B er C dans un " récit de vie " cohérent et unique. Pour lui, comme pour Anna McMullan, les récurrences du dramaricule questionnent la pertinence conceptuelle des oppositions comme différence - identité, intérieur - exréricur, fini - infini, passé - présent, etc. Voir CONNOR Sreven, Samuel Beckett. Repetition, Iheory and suit nous regarderons de plus près quelques récurrences textuelles qui ont échappé à la critique pour préciser la manière dont elles convoquent simultanément une " mémoire intratextuelle " et une " mémoire d'autotraduction ".

Discutant les répétitions intratextuelles de That Time Steven Connor écrit:

Phrases recur insistently, not only within individual sequences, but across sequences, sometimes in close proximity. [...] [M]ost of the motifs are shared between two of the voices alone, giving the sense of asymmetry, or incompleteness. The listener or reader is reminded of words and phrases by repetition and encouraged to form groupings; but these groupings always form the unstable ratio of 2:1, leaving out one of the sequences. This ratio seems to be confirmed by the French version of the play, in which, although the actual words and phrases which are repeated are different from the English, and are also distributed differently, each repeated word or phrase is still usually shared between two speakers only ${ }^{32}$

Connor a raison d'observer que les récurrences textuelles de Cette fois et That Time ne sont pas identiques, ce qui veut dire que les deux versions du dramaticule configurent délibérément, suite au processus d'autotraduction, des " mémoires intratextuelles " différentes. En comparant Cette fois avec That Time (et Damals) on constate que l'autotraduction en français œuvre en effet à réorganiser et parfois à multiplier les " traces intratextuelles" de la pièce.

L'autotraduction qui donne "et de là à pied " pour " and on from there " $\left(A_{1}\right)$ et "à pied donc à la fin " pour «so foot it up in the end" $\left(A_{6}\right)$ établit à travers la répétition de "à pied " des liens intratextuels beaucoup plus directs entre $A_{1}$ et $A_{6}$ qui font que les deux fragments s'évoquent l'un l'autre plus promptement en français qu'en anglais. La situation est similaire pour l'autotraduction constante de " in [out of the rain / out of the cold / off the street]" par "à l'abri [de la pluie / du froid / de la rue]" $\left(\mathrm{C}_{1}, \mathrm{C}_{8}, \mathrm{C}_{9}, \mathrm{C}_{11}\right)$, d'une part, et du choix adoxal qui équivaut "great thing free culture far from home " à "vive la culture gratuite providence des sans-abri " $\left(\mathrm{C}_{8}\right)$ d'autre part ${ }^{33}$. La réitération multiple de " à l'abri » et l'occurrence unique de "sans-abri " dans les bribes de voix $C$ citées sont reliées immédiatement par une "mémoire intratextuelle " qui n'opère pas de la même manière dans That Time. L'autotraduction adoxale de "all bustle Christmas bustle" par " tout ébullition fin d'année " $\left(\mathrm{C}_{0}\right)$ inscrit dans Cette fois au lieu de "Noël " une "fin

Text, op. cit., p. 136-137 et MCMULLAN Anna, Theatre on Trial. Samuel Beckett's Later Drama New York et Londres, Routledge, 1993, chapitre 2, "Masquerades of Self ", p. 52.

32. CONNOR Steven, Samuel Beckett. Repetition, Theory and Text, op. cit., p. 136 et la note 21, p. 211.

33. Un choix d'(auto)traduction adoxal s'éloigne des "normes du traduire attendues". Voi

OUSTINOFF Michaël, Bilinguisme d'écriture et auto-traduction. Julien Green, Samuel Beckett, Vladimir Nabokov, Paris, L'Harmattan, 2001, p. 168. 
d'année " consistante avec les autres "fins " et " années " qui sont variées à loisir dans ce fragment et se convoquent réciproquement: " hiver sans fin à longueur d'année comme si elle ne pouvait pas finir l'année finissante".

Ces trois exemples ("à pied ", "à l'abri " - "sans-abri " et "fin d'année ") peuvent induire la fausse impression que l'autotraduction modifie les " connexions mnésiques " de Cette fois, par rapport à That Time, seulement à l'intérieur de chacune des trois bribes de voix. L'exemple suivant fait voir que ce n'est pas le cas. "To hell lout of there / out of it] " $\left(\mathrm{C}_{1}, \mathrm{C}_{2}, \mathrm{~A}_{7}, \mathrm{~A}_{12}\right)$ et " not a curse [for the old scenes the old names / for the passers] " $\left(A_{2}, A_{5}, A_{7}, A_{10}, A_{12}\right)$ sont autotraduits constamment en français par " au diable ": " au diable [loin de là / loin de tout] " et " au diable [les vieux lieux les vieux noms / les passants] " respectivement. ${ }^{34}$ Ces choix d'autotraduction mettent directement en rapport beaucoup plus de bribes de voix dans Cette fois que dans That Time (notamment $C_{1}, A_{2}, C_{2}, A_{5}, A_{7}, A_{10}, A_{12}$ ) et agencent certaines d'entre elles de manière beaucoup plus cohésive autour des répétitions (il y a ainsi deux " au diable " dans $\mathrm{A}_{7}$ et trois dans $\mathrm{A}_{12}$ ). La " mémoire intratextuelle" de Cette fois associe ainsi " les vieux lieux les vieux noms" de $\mathrm{A}$ avec le Musée des Portraits de $\mathrm{C}$ et invite le lecteur/le spectateur à comprendre celui-ci en termes de ceux-là et inversement.

Cet exemple illustre la "mémoire intratextuelle " différentielle de That Time et Cette fois aussi à travers la récurrence de certains mots qui apparaissent dans l'environnement immédiat de " to hell", "not a curse " et " au diable ". Les "scenes " de " not a curse for the old scenes the old names" $\left(A_{2}, A_{5}, A_{7}, A_{12}\right)$ rappellent immédiatement, en anglais, par l'usage du même mot, les diverses "scènes " que " tu " invente dans $\mathrm{B}\left(\mathrm{B}_{2}, \mathrm{~B}_{3}, \mathrm{~B}_{7}, \mathrm{~B}_{9}, \mathrm{~B}_{10}\right)$ pour " contenir le vide ": celle des deux amoureux sur la pierre au soleil $\left(B_{2}, B_{7}\right)$, celles du ventre maternel et du vieux Chinois $\left(\mathrm{B}_{3}\right)$, celles de la pierre au soleil, du halage et des dunes où " tu " est soit accompagné, soit seul $\left(\mathrm{B}_{q}\right)$ et surtout, en raison de l'environnement restreint de l'adjectif qualificatif, la "vieille scène " (" the old scene ") générique de $\mathrm{B}_{10}$ dont les autres ne sont que des particularisations spatiales et temporelles.

34. L'(auto)traduction allemande garde les deux séries de récurrences intratextuelles distinctes, en offrant pour " $\mathrm{t} 0$ hell lout of there / out of it]" $\left(\mathrm{C}_{1}, \mathrm{C}_{2}, \mathrm{~A}_{7}\right.$ et $\left./ \mathrm{A}_{12}\right)$ " verdammt [raus da /raus aus alldem]" et pour " not a curse [for the old scenes the old names / for the passers]" $\left(A_{2}, A_{5}, A_{7}, A_{11}\right.$ ', $A_{12}$ ) "keinen Pfiff ( fuir die alten Szenen die alten Namen / fuir die Passanten ". Becketr a corrigé la traduction allemande d'Elmar Tophoven de $\mathrm{A}_{7}$, de " verdammt weg von dort " à " verdammt raus da ", de manière à garder consistantes les " mémoires intratexruelles" de Damals er That Time. Cetre correction appuie l'idée que les récurrences des textes becketriens en général et de That Time (Damals, Cette fois) en particulier ne sont pas accidentelles.
Cette fois, en traduisant "scene(s) " par " scène(s)", ne met pas en rapport $\mathrm{B}_{2}$, $\mathrm{B}_{3}, \mathrm{~B}_{7}, \mathrm{~B}_{9}, \mathrm{~B}_{10}$ avec les « lieux " de " au diable les vieux lieux les vieux noms" $\left(\mathrm{A}_{2}\right.$, $A_{5}, A_{7}, A_{12}$ ) à l'instar de That Time. Les " (vieux) lieux" de Cette fois forment en échange un "réseau mnésique intratextuel " avec les " lieux " de $\mathrm{C}_{8}, \mathrm{C}_{10}, \mathrm{~A}_{11}, \mathrm{C}_{11}$ et $\mathrm{C}_{12}$ qui désignent le bureau de poste, la bibliothèque municipale et le pas de la porte et qui autotraduisent dans la quasi majorité des cas l'anglais " place " 35 . L’anglais "place(s)" - qui apparaît également dans $\mathrm{A}_{1}, \mathrm{C}_{2}, \mathrm{C}_{8}, \mathrm{~A}_{8}$ (où il lui arrive de s'appliquer, comme dans $\mathrm{A}_{8}$, à un des "lieux " de Cette fois, à savoir le pas de la porte de $\mathrm{A}_{11}$ ) - n'y est toutefois pas autotraduit par " lieu(x) " mais par d'autres choix qui établissent dans Cette fois d'autres " traces mémorielles intratextuelles».

S'il est peut-être juste de dire, comme Connor, que chaque version a sa propre " mémoire intratextuelle " qui semble favoriser d'emblée des rapports asymétriques entre deux bribes de voix, l'analyse plus détaillée des répétitions dans cet exemple de That Time (Damals) et Cette fois permet toutefois de nuancer, voire contester, cette observation. Les différents éléments réitérés du syntagme "not a curse for the old scenes the old names", à savoir "not a curse" et «scene(s) ", configurent dans That Time une " mémoire " commune de $\mathrm{A}$ et $\mathrm{B}\left(\mathrm{A}_{2}, \mathrm{~B}_{2}, \mathrm{~B}_{3}, \mathrm{~A}_{5}, \mathrm{~B}_{7}, \mathrm{~A}_{7}, \mathrm{~B}_{9}, \mathrm{~B}_{10}\right.$, $A_{12}$ ), tandis que les différents éléments réitérés du syntagme " au diable les vieux lieux les vieux noms ", à savoir " au diable " et " lieu $(\mathrm{x})$ " configurent dans Cette fois une " mémoire " commune de $A$ et $C\left(C_{1}, A_{2}, C_{2}, A_{5}, A_{7}, C_{8}, A_{10}, C_{10}, A_{11}, C_{11}\right.$, $\left.A_{12}, C_{12}\right)$. Si on regarde That Time et Cette fois indépendamment l'un de l'autre, les choses se passent en effet ainsi.

Encore est-il que si l'on prend en considération le fait que Cette fois est, en fin de compte, une autotraduction de That Time, autrement dit que les versions anglaise et française du dramaticule ne sont pas complètement isolées mais entretiennent nécessairement des rapports mutuels, c'est leur interaction même - dont "not a curse for the old scenes the old names" $\leftrightarrow$ " au diable les vieux lieux les vieux noms " n'est qu'un exemple - qui finit par mettre en rapport toutes les trois bribes de voix $\mathrm{A}, \mathrm{B}$ et $\mathrm{C}\left(\mathrm{C}_{1}, \mathrm{~A}_{2}, \mathrm{C}_{2}, \mathrm{~B}_{2}, \mathrm{~B}_{3}, \mathrm{~A}_{5}, \mathrm{~B}_{7}, \mathrm{~A}_{7}, \mathrm{C}_{8}, \mathrm{~B}_{9}, \mathrm{~B}_{10}, \mathrm{~A}_{10}, \mathrm{C}_{10}, \mathrm{~A}_{11}, \mathrm{C}_{11}\right.$ $A_{12}, C_{12}$, dans notre cas). Ceci arrive non seulement grâce aux correspondances respectives de "not a curse " $\leftrightarrow$ " au diable " et "scenes " $\leftrightarrow$ " lieux ", mais aussi grâce aux correspondances de " to hell" $\leftrightarrow$ " au diable ", "place $(s)$ " $\leftrightarrow$ " lieu $(\mathrm{x})$ ", " (in the) place" $\left(\mathrm{A}_{1}\right) \leftrightarrow$ "à perte de vue " $\left(\mathrm{A}_{1}, \mathrm{~B}_{1}, \mathrm{~B}_{3}\right.$ et $\left.\mathrm{B}_{8}\right)$, etc. Ainsi, l'autotraduction qui opère vraisemblablement de manière à distinguer les " mémoires intratextuelles " de Cette fois et That Time inscrit en réalité entre les deux versions

35. Seulement dans $A_{11}$ "lien " autorraduit la conjonction " where ": "forgetting it all where you were and what for", "oubliant tout le lieu où tu étais et pourquoi". 
du dramaticule, au travers de leur interaction, des "traces mémorielles " inédites qui s'évoquent réciproquement et qu'aucune des deux versions, considérée indépendamment, ne saurait activer.

Le regroupement des répétitions intratextuelles auquel le lecteur / le spectateur de Cette fois est invité à procéder n'aboutit pas toujours, même en lecture / spectature unilingue, "à la ratio instable 2:1 qui laisse toujours de côté l'une ou l'autre bribe de voix ". "Inventer " et "invention ", termes de la même famille lexicale, sont distribués équitablement dans Cette fois, dans le sens où chacune des trois bribes $\mathrm{A}, \mathrm{B}$ et $\mathrm{C}$ en contient, dans l'ensemble, non pas une, mais au moins deux occurrences (voir $A_{4}, B_{4}, C_{7}, B_{0}, A_{11}$ ). La seule occurrence dans $A_{4}$ (" tout à tes inventions") s'applique à l'enfant qui, " dix onze ans sur une pierre au milieu des orties géantes ", porte des "conversations imaginaires " avec lui-même sur plusieurs voix ${ }^{36}$. $\mathrm{B}_{4}$ contient pareillement une occurrence unique d' $\mathrm{d}^{\text {' inventer " }}$ qui suggère que la "scène " avec les amoureux sur une pierre à l'orée du petit bois n'est "qu'une de ces histoires que tu allais inventant pour contenir le vide ". La double occurrence d'" inventer " dans $C_{7}$ traite de fictives " l'éternelle vadrouille " et les expériences identitaires de " $\mathrm{tu}$ " ( " l'éternelle vadrouille cherchant à l'inventer ainsi à t $t$ 'inventer ainsi au fur et à mesure "). La seule occurrence de $B_{9}$ met en doute non seulement la réalité passée de la compagne de " tu " (comme $\mathrm{B}_{4}$ ), donc cette scène-là particulière, mais toutes les "scènes " indifféremment de leur cadre spatio-temporel et de la présence ou l'absence des protagonistes (" ou seul dans les mêmes scènes l'inventant ainsi histoire de tenir contenir le vide [...] sur la pierre $[\ldots]$ ou sur le halage $[\ldots]$ jusqu'à ce que [le soleil] meure disparaisse et toi avec tout avec »). Finalement $A_{11}$ contient pas moins de trois occurrences d'" inventer » ( inventant toute l'histoire au fur et à mesure affalé sur le pas de la porte t'inventant toi te réinventant pour la millionième fois ") qui jettent le doute sur la réalité du " retour " raté, " cette dernière fois ", à la " ruine de l'enfant ".

La "mémoire intratextuelle " que configure la récurrence d' inventer ", " invention" et plusieurs mots et syntagmes dans leur environnement restreint, érige un cadre interprétatif qui favorise, à la fois sémantiquement et textuellement, la compréhension de la " mémoire individuelle " du "Souvenant " non pas (seulement, ni tout d'abord) comme un processus de présentification d'un vécu expérientiel passé, mais (aussi, voire principalement) comme le résultat d'un processus de création artistique. Les répétitions de " marmonner " et " renoncer "

36. I.a critique a déjà remarqué le fonctionnement autoréférentiel de $\mathrm{A}_{4}$ qui décrit une situation similaire à celle du "Souvenant ", à la différence près que celui-ci ne " graille " pas " rantôt une voix tantôt une autre jusqu’à en avoir la gorgè en feu ", mais les écoute. dans $A, B$ et $C$ (voir $C_{4}, B_{5}, C_{7}, A_{10}$ et $C_{7}, A_{7}, B_{12}$ respectivement) - parfois plusieurs fois par fragment d'enregistrement - témoignent elles aussi du fait que les « traces mémorielles intratextuelles » de Cette fois ne se limitent pas à une paire donnée de bribes de voix ( $\mathrm{A}$ et $\mathrm{B}, \mathrm{A}$ et $\mathrm{C}$ ou $\mathrm{B}$ et $\mathrm{C}$ ), mais les relient toutes les trois. Il convient aussi de noter que, dans Cette fois, "inventer ", "marmonner " et « renoncer " relèvent d'une isotopie commune qui désigne les divers aspects (cognitifs et expressifs) et étapes (développementales et finales) d'une activité génératrice d'" histoires ", "fables " et autres " scènes ", dont " tu " est simultanément et le sujet et l'objet. $\mathrm{C}_{7}$, qui questionne le plus explicitement l'existence subjective de " tu " et donc nécessairement aussi toute "mémoire individuelle » qu'on serait enclin à lui attribuer, est la seule bribe de voix à actualiser en même temps tous les trois verbes de cette isotopie de la création.

Les itérations lexicales, syntagmatiques et morphosyntaxiques de Cette fois mettent en place une "mémoire intratextuelle " qui se signale par son caractère différentiel autant que par son (soi-disant) rabâchage. Contre le " fond " de l'usage quasi-généralisé de la deuxième personne - qui, comme le dira plus tard Compagnie, est « le fait de la voix ${ }^{37}$ "- les quelques occurrences explicites à la troisième personne et implicites à la première ressortent comme " figures " singulières qui disloquent la régularité, voire la monotonie, des « souvenirs " grammaticaux du lecteur / spectateur du dramaticule ${ }^{38}$. L'autotraduction joue un rôle important dans ce contexte aussi, puisque la fréquence et la nature des indices personnels (pronoms, déterminants et verbes) de Cette fois ne sont point identiques à celles de That Time, tout comme les indices temporels des deux versions de la pièce ne sont pas identiques non plus ${ }^{39}$.

37. "L'emploi de la deuxième personne est le fait de la voix. Celui de la troisième celui de l'autre. Si lui pouvait parler à qui et de qui parle la voix il y aurait une première. "BECKETT Samuel, Compagnie, Paris, Éditions de Minuir, 1985, p. 8.

38. Tout $A_{4}$ est rédigé à la troisième personne, en remplaçant "ton livre d'images " $\left(A_{3}\right)$ par " son livre " er " quand ça te prenait [...] les autres qui batraient les chemins à ta recherche " $\left(\mathrm{A}_{4}\right)$ par "quand ça lui prenait [...] les autres qui battaient les chemins à sa recherche ". "Allons " $\left(\mathrm{C}_{5}\right)$ et "disons" $\left(\mathrm{A}_{7}\right)$, bien qu'il s'agisse d'embrayeurs discursifs presque lexicalisés, continuent à présupposer un " nous" qui est constitué, dans le cas de Cette fois, d'un " je " (la source de la voix?) et le " tu " actualisé de manière constante tout au long du dramaticule.

39. "The old green greatcoat" $\left(C_{2}, A_{8}, A_{12}\right)$ et " the old green holeproof coat " $\left(C_{8}\right)$ deviennent invariablement "ton vieux manteau vert "; "something the dust said " $\left(\mathrm{C}_{11}\right)$ donne "quelque chose que la poussière $t$ 'a dit "; tandis que " and on to the next " $\left(\mathrm{C}_{2}\right)$ correspond à " $t$ 'affaler ailleurs ". conformément à l'autotraduction de " and sat down" $\left(\mathrm{C}_{1}\right)$ par " $t$ 'affaler ». Ce dernier exemple illustre aussi la substitution assez fréquente des modes verbaux personnels de That Time par des formes impersonnelles ou averbales dans (ette fois. Voir par exemple " took the eleven " $\left(A_{11}\right)$, 
Contre le "fond "sémantique largement redondant des "scènes " décrites dans $\mathrm{A}, \mathrm{B}$ et $\mathrm{C}^{40}$, la variation, la nouveauté et même la constance textuelle des mots, syntagmes et phrases se détachent très souvent comme " figure " dans Cette fois. S'il est difficile au lecteur du dramaticule, et plus encore à son spectateur, de garder des "souvenirs " thématiques distincts et précis de chacune des interventions d'une seule et même bribe de voix ( $A, B$ ou $C$ ), il est presque certain que des " faits" textuels, aussi bien itératifs que ponctuels, laissent des " empreintes " dans sa mémoire. Des expressions comme " rails [tout] rouillés " $\left(A_{2}\right)$, "Folie Fourier " $\left(A_{3}, A_{11}\right)$, « blés blondissants " $\left(B_{1}, B_{3}, B_{10}\right)$, " pierre plate " $\left(A_{10}\right)$, " nuit noire [ou clair de lune] " $\left(A_{4}, A_{9}, A_{10}, B_{12}\right)$, « amour à peine un murmure " $\left(B_{1}, B_{2}, B_{3}\right)$, etc. relèvent d'une " mémoire intratextuelle" non seulement sémantique, mais aussi phonétique, par le biais de l'allitération ${ }^{41}$. "Livre d'images " $\left(A_{3}\right)$, " essieu " $\left(B_{6}\right)$, " haltère " $\left(B_{8}\right)$, " musaraigne " $\left(B_{12}\right)$, etc. sont eux aussi susceptibles d'inscrire des " traces mnésiques " dans Cette fois non pas en tant qu'éléments largement récurrents, mais comme occurrences exceptionnelles, voire uniques, dans une œuvre ou presque tout est répété. Dans le cas des termes répétés souvent (comme " orties " dans $A_{3}, A_{4}, A_{0}, A_{10}$ ), c'est la différence d'environnement restreint (parfois " orties géantes", parfois simplement " orties") qui attire l'attention et est retenue.

La dernière section de cet article montrera que la "mémoire intratextuelle " de Cette fois - qui fonctionne de manière complexe, à des multiples niveaux et qui est conditionnée par les " mémoires scénographique " et "d'autotraduction " du dramaticule tout en les conditionnant à son tour - opère souvent aussi autotextuellement et / ou intertextuellement.

"aver le 11 "; " slipped in when no one was looking " $\left(C_{1}\right)$, "guetté le moment de te faufiler "; "to vow you loved each other" $\left(B_{2}\right)$, "pour vous jurer amour"; etc. On retrouve un exemple de changement verbal temporel entre les deux versions, anglaise et française, dans $\mathrm{A}_{11}$ : "the child's ruin you came to look was it still there ", "la ruine de l'enfant que tu étais retourné voir si elle était toujours là ". Cer exemple illustre en même remps une variation temporelle, voire un recul dans le temps, à l'intérieur de Cette fois aussi, puisque dans $A_{1}, A_{2}, A_{5}, B_{11}$ il s'agit de " cette fois où tu es retourné». Pour la différence d'autres marqueurs temporels (noms, adverbes et prépositions) dans That Time et Cette fois voir CONNOR Steven, Samuel Beckett. Repetition, Theory and Text, op. cit., p. 137-138.

40. Grossman parle de cetre invariabilité sémantique / thémarique en termes de "leimouvs " spécifiques à chacune des bribes de voix A, B et C. Voir GROSSMAN Fvelyne, "À la limite... ", op. cit., p. 115-116.

41. "Foley's Folly " qui devient en allemand, suıte à la correcrion de Beckert, "Tuohys ' Tuskulum " confirme le souci d'icération phonérique dans le dramaticule.

\section{La " mémoire autotextuelle"}

\section{et la " mémoire intertextuelle" de Cette fois}

Entendue comme "l'intertextualité interne à l'ensemble des œuvres d'un même auteur ${ }^{42}$ ", l'autotextualité est un phénomène définitoire du corpus beckettien dont la critique a déjà érudié les procédés de "répétition narrative " et " réduplication thématique ${ }^{4.3}$ ". Au sujet même de Cette fois $\mathrm{S}$. E. Gontarski a remarqué:

Like much of the Later Beckett, That Time appears to be an assemblage of reordered fragments from earlier work. Beckett is fond of echoing and alluding to Beckett, and in the earliest version of That Time he self-mockingly described a character as "something out of Beckett". To those familiar with the Beckett world, That Time offers few surprises.

[...] Both stage image and narrative motifs echo a number of earlier works: Krapp's Last

Tape, $[\ldots]$ Play, Nor $I,[\ldots]$ The Unnamable, $[\ldots]$ How It Is $[\ldots]^{44}$.

Les "images scéniques ", les "motifs narratifs" et les " thèmes " ne son toutefois pas les seuls éléments à être réitérés dans le corpus beckettien de manière à mettre en place des "traces mémorielles autotextuelles " qui se convoquent mutuellement à travers genres littéraires, époques de création et langues de rédaction et d'autotraduction. Comme dans le cas de la "mémoire intratextuelle ", c'est la répétition identique ou légèrement variée des mêmes mots, syntagmes et phrases qui contribue autant sinon plus que les contenus sémantiques / thématiques et narratifs à établir des liens directs et concrets entre les diverses créations de Beckett.

En ce qui concerne l'intertextualité, cet autre phénomène définitoire de l'œuvre de l'auteur, son fonctionnement "mémoriel " distinctement textuel - c'est-à-dire lexical, syntagmatique et phrastique - n'a été prouvé de manière systématique et concluante que récemment, même s'il a été reconnu en passant depuis longtemps ${ }^{45}$. Le plus souvent toutefois, et par rapport à Cette fois y compris,

42. DEBRAY-GENETTE Raymonde, "Génétique ex poétique: le cas de Flaubert ", in DEBRAYGENETTE Raymonde et al., Essais de critique génétique, Paris, Flammarion, 1979, p. 23-67, p. 33

43. Angela MOORJANI utilise ces expressions dans son ouvrage Abysmal Games in the Novels of Samuel Beckett, Chapel Hill, University of North Carolina Department of Romance Languages, 1982.

44. GONTARSKI Sranley E., " "Making yourself all up again" in That Time", op. cit., p. 150

45. John Fletcher note déjà en 1967 que "Beckett has borrowed both his [Dante's] idea and his terms from the Divine Comedy" (FLETCHER John, Samuel Beckett's Art, Londres, Chatto \& Windus, 1967, p. 119 , nous soulignons). Les travaux de ACKERLEY C. J., " Demented Particulars: The Annotated Murphy", Journal of Beckett Studies, vol. 7, $n^{\circ} 1$ et 2, automne 1997/printemps 1998, p. 1-215; idem, "Obscure Locks, Simple Keys: The Annotated Watt", Journal of Beckett Studies, vol. 14, $\mathrm{n}^{\circ} 1$ ex 2, automne 2004 / printemps 2005, p. 1-292; CASElli Daniela, Beckett's Dantes, Manchester et New York, Manchester University Press, 2005; er PILLING John, "The 
les " traces mémorielles autotextuelles et intertextuelles " qui traversent le corpus beckettien continuent à être étudiées au seul niveau du signifié ${ }^{46}$. Les quelques exemples suivants veulent faire voir que pour comprendre l'autotextualité et l'intertextualité de Cette fois, il ne suffit pas de connaître "le monde de Beckett " "the Beckett world "), mais il faut encore être familier avec " the Beckett words".

Comme nous l'avons déjà brièvement noté à la fin de la section précédente, le syntagme " nuit noire ou clair de lune " revient constamment dans Cette fois ( $A_{4}$, $\left.\mathrm{A}_{0}, \mathrm{~A}_{10}, \mathrm{~B}_{12}\right)$, en s'imposant à la " mémoire intratextuelle " du lecteur / spectateur à la fois par sa répétition verbatim et par l'allitération en $[\mathrm{n}]$ de "nuit noire ". L'autotraduction joue un rôle essentiel à configurer "nuit noire ou clair de lune " identiquement en français, et à rendre ainsi cette expression plus "mémorable ", puisque les syntagmes de That Time auxquels elle correspond ne sont pas parfaitement identiques, mais légèrement différents: "in the black dark or moonlight " $\left(A_{4}\right)$; " the moonlight " $\left(A_{0}\right)$; " in the dark or moonlight " $\left(A_{10}\right)$; et " in the dark or moonlight" $\left(\mathrm{B}_{12}\right)$.

"Nuit noire ou clair de lune ", qui témoigne d'un travail d'harmonisation intratextuelle beaucoup plus consistante dans Cette fois que dans That Time, relève également d'une "mémoire autotextuelle ", puisque la tournure apparaît presque mot à mot dans une autre autotraduction beckettienne - à savoir Immobile, version française de Still - qui date d'une époque précédant de peu Cette fois: "Tout immobile donc à nouveau calme parfait en apparence yeux fermés lesquels pour anticiper une fois rouverts à moins de trop tarder alors nuit noire ou bien clair de lune ou étoiles ou les deux ${ }^{47}$. "Cette phrase d'Immobile contient deux autres

Annotated Dream of Fair to Middling Women ", Journal of Beckett Studies, vol. 12, $\mathrm{n}^{\circ} 1$ et 2 , automne 2002 / printemps 2003, p. 1-370 - prouvent définitivement que Beckett retient, de ses diverses et nombreuses lectures, des mots, expressions et phrases qu'il inclut par la suire dans ses propres écrits.

46. C'est principalement de cette façon qu'Oliver Vogel étudie les rapports intertexcuels de Cette fois avec la Recherche proustienne; James Acherson ceux avec le fameux poème de Wordsworrh Lines Written a Few Miles above Tintern Abbey; Enoch Brater ceux avec les Sonnets XLIX et $L X X I I I$ de Shakespeare; et Damien Love ceux avec Fragment von Hyperion de Hölderlin. Voir VOGE.L Oliver, "...till that time came... Marcel Proust und Samuel Beckett ", Neute Rundschaut, 106 ${ }^{\mathrm{c}}$ année, no. 1, 1995, p. 154-167; ACHERSON James, Samuel Beckett's Artistic Theory and Practice. Criticism, Drama and Early Fiction, Houndmills, Basingstoke, Hampshire et Londres, MacMillan Press, 1997, chapitre 9 "The Shape of Ideas: That Time ", p. 182-194; BRATER Enoch, op. cit.; et LOVE Damian, "Dic exzentrische Bahn: Samuel Beckett and the Madness of Hölderlin ", Archiv für das Studium der neueren Sprachen und Literaturen, vol. 243, $1^{\circ} 2$, $158^{\mathrm{e}}$ année, 2006, p. 348-365.

47. La " foirade "Still, que Beckett a rédigée du 17 juin au 26 juillet 1972, a été publiée en édition princeps en 1974, Lautotraduction Immobile est postérieure à juillet 1975. Voir COHN Ruby, syntagmes qui sont réitérés tels quels plusieurs fois dans Cette fois, notamment "tout immobile $(s)$ " $\left(\mathrm{B}_{1}, \mathrm{~B}_{2}, \mathrm{~B}_{8}\right)$ et " yeux fermés" $\left(\mathrm{B}_{1}, \mathrm{~B}_{3}, \mathrm{~B}_{7}, \mathrm{~A}_{10}\right)^{48}$. Comme plusieurs "nuit noire ou clair de lune" de Cette fois, " nuit noire ou bien clair de lune " d'Immobile est le résultat d'une autotraduction adoxale de "So quite still again then all quite quiet apparently eyes closed which to anticipate when they open again if they do in time then dark or some degree of starlight or moonlight or both ${ }^{49}$. "A part " nuit noire ou [bien] clair de lune ", "tout immobile(s)" et "yeux fermés", Cette fois et Immobile sont, sauf erreur de notre part, les seuls textes beckettiens à comprendre l'expression " feux du couchant ${ }^{50}$ " $\left(\mathrm{B}_{9}\right)$.

L'ensemble de ces indices pointe vers une " mémoire autotextuelle " que Cette fois partage lexicalement avec Immobile, surtout par le biais de la bribe de voix B. Cette autorextualité lexicale est renforcée thématiquement par le fait que le protagoniste innommé d'Immobile se trouve lui aussi "à la fenêtre " parfois "jusqu'à nuit noire " comme "tu " dans la " scène "-cadre de B ("à la fenêtre dans le noir " $\left.-B_{4}, B_{12}\right)^{51}$. Ce protagoniste ne fait en apparence la plupart du temps " aucun autre mouvement [d']aucune sorte " qu'ouvrir et fermer les yeux. Ouvrir et fermer les yeux sont des mouvements de prédilection de toutes les figures humaines dans Cette fois aussi.

L'exemple de "nuit noire ou clair de lune " montre que certaines répétitions lexicales, syntagmatiques et/ou phrastiques de Cette fois inscrivent simultanément dans le dramaticule les " traces " d'une " mémoire intratextuelle ", d'une " mémoire autotextuelle " et d'une "mémoire d'autotraduction " - qui a en vue non seulement le passage de That Time à Cette fois, mais aussi celui antérieur de

A Beckett Canon, Ann Arbor, University of Michigan Press, 2001, p. 319 er PilLing John A Samuel Beckett Chronology, op. cit., p. 197. Nous urilisons l'édition d'Immobile référencée dans la Bibliographie er puisqu'il s'agit d'un texte courr, nous ne donnons pas d'indications de page.

48. " Immobile " apparaît aussi deux fois dans $\mathrm{B}_{\text {, }}$, dans l'expression " immobiles comme marbre ". "Tout immobile " et " yeux fermés" se retrouvent ensemble dans $B_{1}$. Cette fois contient trois occurrences de " tout inmmobile(s) ", tandis qu'Immobile n'en contient pas moins de neuf.

49. BECKETT Samuel, The Complete Short Prose, 1929-1989, édité, préfacé et annoté par Stanley E. GONTARSKl, New York, Grove Press, 1995, p. 241.

50. "Feux du couchant " apparaît dans la troisième phrase d'Immobile: «Clair enfin fin d'une journée sombre le soleil brille enfin er disparaît. Assis immobile face à la fenêtre face à la vallée ici temps normal tourner la tête et le fixer au sud-ouest le soleil qui décline. Voir se lever certains écats et aller se poster à la fenêtre ouest immobile à le fixer le soleil qui décline et ensuite les feux du couchant."

51. La " scène " "à la fenêtre dans le noir " est une "scène "-cadre de la bribe de voix B dans Cette fois dans le sens où elle fonctionne comme une matrice qui englobe, génère er démantèle d'autres " scènes " (" sur la pierre ", " sur le halage ", " dans les dunes "). 
Still à Immobile. La première occurrence de cette expression dans $\mathrm{A}_{4}$ a lieu dans un environnement restreint où au moins deux autres syntagmes sont configurés autotextuellement (parfois) par autotraduction: "gorge en feu ", dont la seule autre occurrence dans le corpus beckettien se retrouve dans D'un ouvrage abandonné et " orties géantes" qui apparaît une fois dans Premier amour et une fois dans Watt.

D'autres tournures et mots de Cette fois - dont plusieurs de ceux qui attirent l'artention du lecteur / spectateur en raison de leur occurrence unique dans le dramaticule - portent les " traces " d'une " mémoire autotextuelle ". Le "livre d'images " de $\mathrm{A}_{3}$ rappelle ainsi le seul autre " livre d'images " du corpus beckettien, à savoir celui de la poupée de Mildred dans l'" histoire " de Winnie dans Oh les beaux jours. L'" essieu " qui fonctionne comme terme de comparaison surprenant dans $B_{6}$ n'a que deux autres occurrences dans tout le corpus: l'une littérale dans Tous ceux qui tombent, l'autre similaire à celle de Cette fois, c'est-à-dire dans une figure décrivant comparativement les rapports entre deux amoureux, dans Comment c'est. ${ }^{52}$ Tous ces exemples - qu'il n'est pas lieu d'étudier en détail ici, mais dont le fonctionnement précis mériterait d'être explicité - parlent en faveur d'une "mémoire autotextuelle" de Cette fois qui conditionne et est en même temps conditionnée par d'autres " mémoires ": " intratextuelle ", "d'autotraduction " et, comme l'exemple suivant le montrera, aussi « intertextuelle ".

Dans son cahier de mise en scène de Damals à Berlin, Beckett a inscrit un bref extrait de Fragment von Hyperion du poète romantique allemand Friedrich Hölderlin, en suggérant ainsi que la bribe de voix $\mathrm{B}$, à laquelle cette citation a trait, évoque non (seulement) des "scènes " existentielles du passé du "Souvenant ", mais (aussi) des " souvenirs" livresques ${ }^{53}$. Certains mots et syntagmes de la bribe de voix $\mathrm{B}$ de Cette fois comme "ciel d'azur " $\left(\mathrm{B}_{3}\right)$ et " feux du couchant " $\left(\mathrm{B}_{9}\right)$, à quoi on peut ajouter " à peine un murmure " $\left(\mathrm{B}_{1}, \mathrm{~B}_{2}, \mathrm{~B}_{3}, \mathrm{~B}_{11}\right)$, " pas un bruit " $\left(B_{1}, C_{2}, B_{2}, C_{11}, C_{12}\right)$, « scène $(s)$ » $\left(B_{2}, B_{3}, B_{7}, B_{9}, B_{10}\right)$, etc. font partie de ce qu'on pourrait appeler un fond linguistique commun au Romantisme français. Victor

52. Voir BECKETT Samuel, D’un ouvrage abandonné, traduit de l'anglais par Ludovic et Agnès JANVIER en collaborarion avec l'auteur, in Têtes-mortes, Paris, Éditions de Minuit, 1972, p. 29; idem, Premier amour, Paris, Éditions de Minuit, 1970, p. 28; idem, Watt, traduit de l'anglais par Ludovic et Agnès JANVIER en collaboration avec l'auteur, Paris, Éditions de Minuir, 1968, p. 161 ; idem, Oh les beaux jours, traduit de l'anglais par l'aureur, in Oh les beaux jours suivi de Pas moi, Paris, Éditions de Minuit, 1974, p. 66; idem, Comment c'est, Paris, Éditions de Minuir, 1961, p. 47.

53. "B. Alles war nun Stille. Wir sprachen kein Wort, wir berïhrten uns nicht, wir saben uns nicht an... Hölderlin. Hyperion-Fragment. " Voir GONTARSKI Stanley E., The Theatrical Notebooks of Samuel Beckett. Volume IV: The Shorter Plays, op. cit., p. 389, 391. Le contexte de la bibliorhèque publique dans la bribe de voix $\mathrm{C}$ renforce l'hypothèse des souvenirs livresques dans Cette fois.
Hugo, représentant prototypique du courant, décline itérativement des "cieux d'azur " et des "feux du couchant ", dont les premiers connotent habituellement un "spectacle [métaphysique, naturel, intersubjectif et subjectif] rassurant ", donc positif, tandis que les deuxièmes ont en revanche, le plus souvent, des connotations opposées et négatives ${ }^{54}$.

La bribe de voix B pointe alors intertextuellement, grâce à des tournures particulières - dont certaines participent aussi d'une " mémoire in tratextuelle ", d'une "mémoire autotextuelle » et/ou d'une "mémoire d'autotraduction " - vers un héritage et un topos romantiques que That Time, Damals et Cette fois actualisent différemment, chaque version du dramaticule par rapport à son propre contexte linguistique, littéraire et culturel: dans That Time on a cru pouvoir identifier les " traces mémorielles » de Wordsworth, dans Damals Beckett lui-même a signalé celles de Hölderlin et dans Cette fois la présence intertextuelle des romantiques français est plutôt facile à retracer ${ }^{55}$.

Cette fois renvoie à travers $\mathrm{B}$ non seulement à un héritage romantique mais aussi à sa réception critique, contemporaine du courant ou bien exercée ultérieurement par des symbolistes comme Mallarmé. Dans le poème Promenade de Sainte-Beuve la voix lyrique du fictif Joseph Delorme s'élève contre Chateaubriand, Lamartine, Nodier et Hugo, en accusant d'artifice leur éloge du sublime. L'expression "blés blondissants" - répétée plusieurs fois dans Cette fois - participe directement de cette attitude anti-sublime du poète dont l'« ennu[i] de vivre » et le " vouloir mourir " est " guéri " non par quelque " spectacle " grandiose de la nature, mais par le menu fait isolé perçu lors d'une habituelle "promenade " solitaire ${ }^{56}$. La critique du sublime de Promenade est fondée, au moins

54. Voir à titre d'exemple, pour "ciel d'azur ", HUGO Victor, "Spectacle rassurant", in CEuvres poétiques, vol. I, Paris, Gallimard, coll. "Bibliorhèque de la Pléiade ", 1964 (recueil Les rayons et les umbres), p. 1062; et pour "feux du couchant ", idem, "La confiance du marquis Fabrice ", in La légende des siècles. La fin de Satan. Dieu, Paris, Gallimard, coll. "Bibliorhèque de la Pléiade ", 1950, p. 315

55. Si cette présence est en effer assez facile à retracer, son rôle textuel n’est pas nécessairement évident d'emblée.

56. "Laissons Chareaubriand, loin des traces profanes, / À vingt ans s'élancer en d'immenses savanes, / Un bâton à la main, et ne rien demander / Que d'entendre la foudre en longs éclats gronder, / Ou mugir le lion dans les forêrs superbes [...] / Laissons à Lamarrine, à Nodier, nobles frères, / Leur Jura bien-aimé, tant de scènes contraires / En un même horizon, et des blés blondissants, / Et des pampres jaunis, er des bœufs mugissants, [...] / Qu'aussi Victor Hugo, sous son donjon qui croule, / Er le Rhin à ses pieds, inrerroge er déroule / Les souvenirs des lieux [...] / Que du Heuve qui passe il écoute les voix, / Er que le grand vieillard lui parle d'autrefois! / Bien; il faut l'aigle aux monts, le géant à l'abyme, / Au sublime spectacle un spectareur 
rhétoriquement, sur l'incapacité avouée du sujet poétique d'être un "spectateur sublime ", car il préfère "reste[r] plus bas ". Comme les "scènes " inventées par la bribe de voix B dans Cette fois, les "scènes " sublimes et non-sublimes de Promenade sont explicitement présentées comme étant une fonction du sujet: " au sublime spectacle un spectateur sublime [Chateaubriand, Lamartine, Nodier et Hugo] ", au spectacle non-sublime un spectateur non-sublime (Sainte-Beuve par le biais de Delorme). Mais si l'impuissance poétique du sujet de Promenade n'est que partielle - il remplace les "scènes " sublimes des autres poètes par ses propres "scènes " non-sublimes -, l'impuissance créatrice de " tu " dans Cette fois est dite totale: les "scènes " intertextuellement autres de B (dont nous avons indiqué seulement celles à "mémoire " simultanément romantique et antiromantique) ne sont pas remplacées par des "scènes " propres, mais disparaissent vraisemblablement toutes dans le " vide".

Encore est-il que la "scène »-cadre, "à la fenêtre dans le noir ", celle-là même qui contient toutes les autres et raconte leur disparition, leur " survit » ultimement et dément ainsi par son existence textuelle même, ses propres "dires". Cette " scène " post-« scène " ou bien "scène " malgré elle relève des " traces mémorielles intertextuelles " symboliques et plus précisément mallarméennes. "Le soleil se mourant " de " rien qui bouge sinon l'eau et le soleil se mourant jusqu'à ce qu'il meure disparaisse et toi avec tout avec " $\left(B_{9}\right)$ " rappelle " directement le poème L'Azur de Stéphane Mallarmé auquel Beckett a fait allusion déjà dans Dream of Fair to Middling Women ${ }^{57}$. Dans L'Azur, comme dans Promenade et comme dans la "scène "-cadre de B dans Cette fois, il s'agit de l'impuissance créatrice du sujet. Le " poète impuissant " de Mallarmé, à l'" âme vide " et à la " cervelle vidée " (voir la " tête vide " de $\mathrm{B}_{4}$ ), " n'a plus l'art d'attifer la sanglotante idée " (voir $\mathrm{B}_{12}$ : " cette dernière fois $[\ldots]$ où tu as essayé tant et plus et n’as pas pu plus moyen plus de mots pour contenir le vide "), raison pour laquelle il veut "fuir " et s'échapper "vers un trépas obscur " (voir $\mathrm{B}_{12}$ : "et le laisser venir [le suaire] et pas plus mal qu'avant vaste suaire venu t'ensevelir et pas plus mal qu'avant ou guère pas plus ou guère ").

sublime. / Moi, j’aime à cheminer er je reste plus bas. "SAINTF-BEUVE, «Promenade ", in Vie. poésies et pensées de Joseph Delorme, Plan de la Tour (Var), Éditions d'aujourd'hui, 1985 [1929], p. 121-123, p. 121-122

57. Voir BECKETT Samuel, Dream of Farr to Middling Women, édité par Foin ()'BRIAN et Edirlı FOURNIER, Londres et Paris, Calder Publications, 1993, p. 31 ; ACKERLEY C. et GONTARSKI Stanley E., The Grove Companion to Samuel Beckett, op. cit., p. 341 ; et MALLARMÉ Sréphane, "L'Azur ", in CEuvres complètes, vol. I, Paris, Gallimard, coll. "Bibliorhèque de la Pléiade ", 1998 (recueil Poésies), p. 14-15.
L'impuissant poète mallarméen se révèle toutefois impuissant jusque dans sa fuite même: "Où fuir? [...] Où fuir dans la révolte inutile et perverse [contre] le désert stérile? ", car il n'y a pas de "nuit hagarde, [de] lambeaux " qu'on puisse " jeter sur [le] mépris navrant » et " la sereine ironie " " de l'éternel azur » idéel qui continue à " hanter " le poète ("Je suis hanté. L'azur! L'azur! L'azur! L'azur!") malgré son impuissance avouée de l'" attifer "-exprimer. Comme dans la bribe de voix B de Cette fois, l'impuissance créatrice du sujet mallarméen relève vraisemblablement d'un manque de moyens expressifs. Chez Mallarmé ces moyens devraient être mis au service de "l'azur »-« idéal cruel " qui subsiste " méchamment victorieux " même dans leur absence, tandis que dans Cette fois les " mots " manquants devraient " contenir le vide " et non un quelconque idéal idéel. Selon les "dires" métadiscursifs respectifs des voix créatrices dans L'Azur et Cette fois, les moyens expressifs dont elles sont dépourvues devraient donc servir des objets différents. Toujours est-il que ces voix ne s'arrêtent pas tout simplement faute de moyens, mais continuent à "dire " leur impuissance de dire. En plus, elles se montrent conscientes du paradoxe qui les sous-tend, autrement dit du décalage entre leur " dire " impuissant et leur " faire " scriptural malgré tout. Le " poète impuissant " de L'Azur produit toute une série d'images de son impuissance en s'exhortant contradictoirement " Encor! ", tandis que la bribe de voix B continue à parler même d'" outre-tombe " ("vaste suaire venu t'ensevelir et pas plus mal qu'avant ou guère pas plus ou guère ") par le biais de multiples " traces mémorielles intertextuelles ».

Dans cet article nous avons montré que Cette fois relève d'une multitude de " mémoires " qui se conditionnent réciproquement. Nous avons détaillé surtout ces « mémoires »-là sur lesquelles la critique ne s'est penchée jusqu'à présent que sporadiquement, à savoir une "mémoire scénique ", une "mémoire intratextuelle ", une " mémoire d'autotraduction ", une " mémoire autotextuelle " et une " mémoire intertextuelle". Toutes ces "mémoires" sont inscrites dans Cette fois directement, par des " traces " textuelles concrètes qui opèrent non seulement au niveau des contenus signifiés, mais aussi à celui des signifiants (mots, syntagmes et phrases) qui s'agencent dans des configurations signifiantes complexes, de même qu'à celui des dimensions médiatiques (verbale, auditive et visuelle) inhérentes au dramaticule en tant que pièce de théâtre.

Nous avons aussi fait voir que les diverses " mémoires » de Cette fois ne sont pas coupées les unes des autres, ni ne fonctionnent isolément, mais interagissen entre elles, en se "rappelant ", déterminant, formant et déformant mutuellement. 
La même " trace " textuelle et/ou médiatique peut ainsi relever simultanément de plusieurs "mémoires », sa particularité consistant précisément dans le fait de les inscrire toutes dans le dramaticule en même temps. Cette fois se découvre ainsi être un excellent exemple non seulement de ce qu'on convient d'appeler le " théâtre de la mémoire " de Beckett (terme qui favorise principalement une "mémoire individuelle "), mais aussi d'une pratique artistique qui explore et exploite à fond une pluralité de « mémoires ». 